\title{
Influence of initial turbulence level on the flow and sound fields of a subsonic jet at a diameter-based Reynolds number of $\mathbf{1 0}^{5}$
}

\author{
C. Bogey ${ }^{1} \dagger$, O. Marsden ${ }^{1}$ and C. Bailly ${ }^{1,2}$ \\ ${ }^{1}$ Laboratoire de Mécanique des Fluides et d'Acoustique, UMR CNRS 5509, Ecole Centrale de Lyon, \\ Université de Lyon, 69134 Ecully CEDEX, France \\ ${ }^{2}$ Institut Universitaire de France, 103 boulevard Saint-Michel, 75005 Paris, France
}

(Received 12 July 2011; revised 24 February 2012; accepted 27 March 2012;

first published online 18 May 2012)

Five isothermal round jets at Mach number $M=0.9$ and Reynolds number $R e_{D}=10^{5}$ originating from a pipe nozzle are computed by large-eddy simulations to investigate the effects of initial turbulence on flow development and noise generation. In the pipe, the boundary layers are untripped in the first case and tripped numerically in the four others in order to obtain, at the exit, mean velocity profiles similar to a Blasius laminar profile of momentum thickness equal to $1.8 \%$ of the jet radius, yielding Reynolds number $R e_{\theta}=900$, and peak turbulence levels $u_{e}^{\prime}$ around $0,3 \%, 6 \%, 9 \%$ or $12 \%$ of the jet velocity $u_{j}$. As the initial turbulence intensity increases, the shear layers develop more slowly with much lower root-mean-square (r.m.s.) fluctuating velocities, and the jet potential cores are longer. Velocity disturbances downstream of the nozzle exit also exhibit different structural characteristics. For low $u_{e}^{\prime} / u_{j}$, they are dominated by the first azimuthal modes $n_{\theta}=0,1$ and 2, and show significant skewness and intermittency. The growth of linear instability waves and a first stage of vortex pairings occur in the shear layers for $u_{e}^{\prime} / u_{j} \leqslant 6 \%$. For higher $u_{e}^{\prime} / u_{j}$, threedimensional features and high azimuthal modes prevail, in particular close to the nozzle exit where the wavenumbers naturally found in turbulent wall-bounded flows clearly appear. Concerning the sound fields, strong broadband components mainly associated with mode $n_{\theta}=1$ are noticed around the pairing frequency for the untripped jet. With rising $u_{e}^{\prime} / u_{j}$, however, they become weaker, and the noise levels decrease asymptotically down to those measured for jets at $R e_{D} \geqslant 5 \times 10^{5}$, which are likely to be initially turbulent and to emit negligible vortex-pairing noise. These results correspond well to experimental observations, made separately for either mixing layers, jet flow or sound fields.

Key words: free shear layers, jet noise, shear layer turbulence

\section{Introduction}

Since the seminal work done by Crow \& Champagne (1971) and Brown \& Roshko (1974) on coherent structures, the effects of the initial conditions in mixing layers and jets have been investigated in a large number of experiments over the past forty 
years. As reported in the review papers written by Crighton (1981), Gutmark \& Ho (1983), Hussain (1983) and Ho \& Huerre (1984), these effects have been found to be strong, and hence recognized as one of the reasons for the discrepancies observed between data in the literature for such flows. The initial conditions of a free shear layer have also been shown to be characterized by three main parameters, namely the Reynolds number $\operatorname{Re}_{\theta}$ based on the shear-layer momentum thickness $\delta_{\theta}$, the (laminar, transitional or turbulent) flow state, and the peak fluctuation intensity with respect to the jet velocity $u_{e}^{\prime} / u_{j}$. On this matter, the reader can refer for instance to the two thorough publications by Hussain \& Zedan $(1978 a, b)$.

The last two parameters mentioned above, i.e. the initial flow state and disturbance levels, are inherently linked. Indeed, in broad outline, the initial state of a shear layer can be divided into two main categories: laminar (or weakly disturbed) states when the peak fluctuation intensity $u_{e}^{\prime} / u_{j}$ is not appreciably higher than $1 \%$, and turbulent (or highly disturbed) states when $u_{e}^{\prime} / u_{j}$ is around $10 \%$. The transition from laminar to turbulent flow takes place in the mixing layer in the first case, but moves to the nozzle boundary layers in the second case, which is very likely to cause significant changes in the turbulent and acoustic features of free shear flows as pointed out by Mollo-Christensen, Kolpin \& Martucelli (1964). These changes have been studied in several experiments, in particular by tripping the boundary layers, thus generating turbulent exit conditions, for jets at moderate diameter-based Reynolds numbers around $R e_{D}=10^{5}$ whose initial state would otherwise be laminar.

The impact of initial disturbances on the development of mixing layers has been described, for example, in the works of Batt (1975), Hussain \& Zedan (1978a,b), Browand \& Latigo (1979), Husain \& Hussain (1979), Hussain \& Husain (1980), and Bell \& Mehta (1990). Mixing layers with laminar upstream conditions have been shown to grow at a higher rate with an overshoot in turbulence intensity around the first stage of vortex merging. The formation and persistence of coherent eddies in shear layers in the presence of free-stream turbulence have also been discussed by Chandrsuda et al. (1978) and Wygnanski et al. (1979). Similarly, the effects of laminar/turbulent initial conditions have been examined for jets. Jets with laminar exit boundary layers have been found to develop more rapidly in the experiments of Hill, Jenkins \& Gilbert (1976), Russ \& Strykowski (1993) and Xu \& Antonia (2002), among others. The influence of inlet velocity fluctuations on the control of jets subjected to tonal excitation has also been explored by Zaman \& Hussain (1980), Zaman (1985a), Lepicovsky \& Brown (1989), Raman, Zaman \& Rice (1989) and Raman, Rice \& Reshotko (1994). Finally, as stated in papers by Lilley (1994) and Harper-Bourne (2010), noise generation in subsonic jets appears to depend strongly on the initial flow state. Additional noise components, attributed to shear-layer vortex pairings, have been obtained for initially laminar jets by Maestrello \& McDaid (1971), Grosche (1974), Zaman (1985b), and Bridges \& Hussain (1987).

The experiments listed above demonstrated the importance of laminar/turbulent initial conditions in free shear flows. In most cases, however, the effects of the turbulence level cannot be clearly distinguished from those of other parameters, such as the shape or the thickness of the boundary layers, which may also vary. Fortunately, some careful investigations have been performed, such as those by Hussain \& Zedan $(1978 a, b)$ dealing with variations in the momentum-thickness Reynolds number $R_{\theta}$ at fixed $u_{e}^{\prime} / u_{j}$ and, conversely, variations in $u_{e}^{\prime} / u_{j}$ at fixed $R e_{\theta}$ for both laminar and turbulent initial shear layers. In the latter case, laminar mean velocity profiles at $R e_{\theta}=200$ with $u_{e}^{\prime} / u_{j}$ between $8.4 \%$ and $17.2 \%$ were in particular considered. 
The present work, in the same way, aims to study the influence of the initial turbulence on subsonic jets at identical Mach and Reynolds numbers, characterized by similar mean velocity profiles but peak turbulence intensities $u_{e}^{\prime} / u_{j}$ ranging from 0 to $12 \%$ at the nozzle exit. It has been made possible thanks to the increase in computer power, and to the rapid progress in the field of computational aeroacoustics since the jet simulations of Freund (2001) and Bogey, Bailly \& Juvé (2003), see the reviews by Bailly \& Bogey (2004), Colonius \& Lele (2004) and Wang, Freund \& Lele (2006) for instance. Indeed, simulations can now be used as numerical experiments under controlled conditions to improve our knowledge on jet flow and acoustics, as illustrated in Bogey \& Bailly (2007) and Bogey et al. (2009a).

This work is the natural continuation of our previous large-eddy simulations (LES) of subsonic jets. Following a preliminary attempt to compute an initially turbulent jet in Bogey, Barré \& Bailly (2008), two studies were carried out for jets at Mach number $M=0.9$ and Reynolds numbers $R_{D}=10^{5}$. In the first study, detailed in Bogey \& Bailly (2010), the jets are initially laminar, leading to strong vortex pairings in the shear layers. In the second one, in Bogey, Marsden \& Bailly (2011a,b), the jets are initially in a highly disturbed, transitional state. More precisely, they are rendered nominally turbulent by applying a trip-like excitation to the boundary layers inside a pipe nozzle. At the exit, Blasius laminar mean velocity profiles of momentum thickness $\delta_{\theta} / r_{0}=1.8 \%$ and peak turbulent intensities $u_{e}^{\prime} / u_{j}=9 \%$ are specified $\left(r_{0}\right.$ is the pipe radius), in agreement with the initial conditions measured in tripped jets by Zaman $(1985 a, b)$. The final LES using a grid of 252 million points is shown to provide shear-layer solutions that are practically grid-converged and, more generally, results that can be regarded as numerically accurate as well as physically relevant. The jet mixing layers, while exhibiting a wide range of turbulent scales, are also found to display attenuated but persistent vortex pairings.

In the present paper, LES of five round isothermal jets at Mach number $M=0.9$ and Reynolds number $R e_{D}=10^{5}$, performed using grids of 252 million points with low-dissipation schemes and relaxation filtering as a subgrid dissipation model, are reported. For all jets, laminar mean velocity profiles of thickness $\delta_{\theta} / r_{0}=1.8 \%$, yielding a Reynolds number $\operatorname{Re}_{\theta}=900$, are imposed inside a pipe nozzle, as in Bogey et al. $(2011 a, b)$. The values of the exit peak turbulence intensities $u_{e}^{\prime} / u_{j}$ are fixed approximately at $0,3 \%, 6 \%, 9 \%$ or $12 \%$, respectively, by numerically tripping the boundary layers in the pipe in the last four cases. The influence of the initial turbulence level on the flow field as well as on the acoustic far field is thus investigated in a systematic manner. Particular attention is paid to the changes in the nozzle-exit flow conditions and in the shear-layer transition as nozzle-exit disturbances gradually increase, and to their consequences on sound sources including pairings of large-scale structures and broadband noise amplification in the mixing layers. For an in-depth description of the structural variations of turbulence with $u_{e}^{\prime} / u_{j}$, mean and r.m.s. values of velocities, integral length scales, spectra, modal distributions in the azimuthal direction, skewness and Kurtosis factors are provided. Comparisons are also made with measurements available in the literature for jets at Reynolds numbers $R e_{D} \geqslant 5 \times 10^{5}$, in order to examine the possible convergence of the results towards typical high-Reynolds-number data as the exit turbulence intensity rises. Note at this point that because of LES limitations using current computational resources, the present study is performed at $R e_{D}=10^{5}$ and $R e_{\theta}=900$ which are both moderate Reynolds numbers. Different results could be expected for practical jets at much higher Reynolds numbers as suggested by recent work in Bogey, Marsden \& Bailly (2012). 
The paper is organized as follows. In $\S 2$, the parameters of the LES and of the extrapolation of the LES near field to the far field, including numerical algorithm, computational grids and times, are presented. In $\S 3$, the jet inflow conditions are first characterized. The shear-layer and jet flow fields as well as the acoustic fields are then shown and analysed. Concluding remarks are given in $\S 4$. The amplification rates of linear instability waves downstream of the jet nozzle exit are finally examined in the Appendix.

\section{Simulation parameters}

\subsection{Jet definition}

Isothermal round jets at Mach number $M=u_{j} / c_{a}=0.9$ and Reynolds number $R e_{D}=u_{j} D / v=10^{5}$ are considered $\left(u_{j}\right.$ is the jet inflow velocity, $c_{a}$ is the ambient speed of sound, $D$ is the nozzle diameter, and $v$ is the kinematic molecular viscosity). They originate from a pipe nozzle of radius $r_{0}=D / 2$ and length $2 r_{0}$. The ambient temperature and pressure are $T_{a}=293 \mathrm{~K}$ and $p_{a}=10^{5} \mathrm{~Pa}$. At the nozzle exit at $z=0$, the width of the nozzle lip is $0.053 r_{0}$. At the pipe inlet at $z=-2 r_{0}$, a laminar Blasius boundary layer of thickness $\delta=0.15 r_{0}$, or equivalently of momentum thickness $\delta_{\theta}=0.018 r_{0}$, yielding a Reynolds number $R e_{\theta}=u_{j} \delta_{\theta} / v=900$, is imposed. This value of $\delta_{\theta} / r_{0}$ corresponds to that measured by Zaman $(1985 a, b)$ in tripped subsonic jets at Reynolds numbers around $10^{5}$. The axial velocity profile is given by a polynomial approximation of the Blasius profile. Radial and azimuthal velocities are initially set to zero, pressure is kept constant at its ambient value, and the temperature is determined by a Crocco-Busemann relation.

In four LES referred to as Jet3\%, Jet6\%, Jet $9 \%$, and Jet $12 \%$, the jet boundary layers are tripped inside the pipe by adding random low-level vortical disturbances decorrelated in the azimuthal direction. The trip-like excitation is applied at $z=-r_{0}$ in the first three simulations and at $z=-0.2 r_{0}$ in the fourth one. The excitation magnitudes are empirically chosen in order to obtain, at the pipe exit, peak turbulence intensities $u_{e}^{\prime} / u_{j}$ around $3 \%, 6 \%, 9 \%$ and $12 \%$ in Jet3\%, Jet6\%, Jet9\%, and Jet12\%, respectively, as well as mean velocity profiles in agreement with the laminar profiles imposed at the pipe inlet, which will be illustrated in $\$ 3.1 .1$. As evidenced by the experiments of Hussain \& Zedan $(1978 a, b)$ and Zaman $(1985 a, b)$ for instance, it is indeed possible to find high levels of velocity fluctuations together with laminar velocity profiles close to the nozzle exit of tripped jets. The simulation Jet9\% corresponds exactly to Jetring1024drdz in Bogey et al. (2011a), where the tripping methodology is detailed, and is shown to generate negligible spurious acoustic waves. In that reference, the very weak sensitivity of the LES results with respect to the tripping procedure is also displayed. In a fifth LES referred to as Jet0\%, no boundarylayer excitation is used in order to consider an initially fully laminar jet. In all simulations, pressure fluctuations of maximum amplitude $200 \mathrm{~Pa}$ random in both space and time are added in the shear layers between $z=0.25 r_{0}$ and $z=4 r_{0}$ from $t=0$ up to non-dimensional time $t=12.5 r_{0} / u_{j}$ in order to speed up the initial transitory period. This also enables turbulent development to be triggered in the Jet0\% configuration. In that case, the jet flow does not relaminarize, which indicates the presence of a feedback mechanism taking place in the pipe or at the nozzle lip. Very small inlet disturbances could have been added in Jet0\%, as was done in Bogey \& Bailly (2010) for a jet at $R e_{D}=10^{5}$ with $\delta=0.05 r_{0}$ at the nozzle exit. The results obtained with or without these disturbances were however found not to differ fundamentally. 


\subsection{LES procedure and numerical methods}

The numerical methodology is identical to that used for recent jet simulations in Bogey \& Bailly (2010) and Bogey et al. (2011a,b). The LES are carried out using a solver of the three-dimensional filtered compressible Navier-Stokes equations in cylindrical coordinates $(r, \theta, z)$ using low-dissipation and low-dispersion finite differences developed in Bogey \& Bailly (2004). The axis singularity is taken into account by the method of Mohseni \& Colonius (2000). Fourth-order eleven-point centred finite differences are used for spatial discretization, and a second-order six-stage low-storage Runge-Kutta algorithm is implemented for time integration. To circumvent the time-step restriction induced by the cylindrical coordinates, the derivatives in the azimuthal direction around the axis are calculated using every $n$th grid point, from $n=2$ up to $n=64$ near the centreline, as described in Bogey, de Cacqueray \& Bailly (2011). To remove grid-to-grid oscillations, a sixth-order elevenpoint centred filter designed in Bogey, de Cacqueray \& Bailly (2009b) to mainly damp the shortest waves discretized is applied explicitly to the flow variables every time step. The discretization at the boundaries is performed using non-centred finite differences and filters provided in Berland et al. (2007) and Bogey \& Bailly (2010).

The explicit filtering is also employed as a subgrid high-order dissipation model to relax turbulent energy from scales at wavenumbers close to the grid cut-off wavenumber while leaving larger scales mostly unaffected, as suggested by Visbal \& Rizzetta (2002). More details on the LES-RF approach based on relaxation filtering are available in Bogey \& Bailly $(2006 a, b, 2009)$. The accuracy of the LES fields in Jet9\% is investigated in Bogey et al. (2011a) based on the transfer functions associated with molecular viscosity, relaxation filtering and time integration. In that LES, molecular viscosity is shown to be the dominant dissipation mechanism for the scales discretized at least by six or seven points per wavelength. The physics of the larger turbulent structures is therefore expected not to be governed by numerical or subgrid-modelling dissipation. The effective flow Reynolds number should also not be artificially decreased, as might be the case when eddy-viscosity LES models are used, as pointed out by Domaradzki \& Yee (2000).

Finally, the non-reflective boundary conditions of Tam \& Dong (1996) are specified, with the addition at the outflow of a sponge zone combining grid stretching and Laplacian filtering in order to gradually damp turbulent fluctuations as proposed in Bogey \& Bailly (2002). The non-reflective conditions are also applied at the pipe inlet.

\subsection{Simulation parameters}

As indicated in table 1, the LES are performed using two grids, one for Jet0\%, another for Jet3\%, Jet6\%, Jet $9 \%$, and Jet $12 \%$, both containing $n_{r} \times n_{\theta} \times n_{z}=$ $256 \times 1024 \times 962=252$ million points. They are identical to the grids employed for the simulations referred to respectively as Jetring $1024 \mathrm{dz}$ and Jetring $1024 \mathrm{drdz}$ in Bogey et al. $(2011 a, b)$. In the grid used for Jet0\%, there are 169 points along the pipe nozzle, 62 points within the jet radius, and 19 points inside the inlet boundary layers. The minimum mesh spacings in the radial, azimuthal and axial directions are $\Delta r / r_{0}=0.0072, r_{0} \Delta \theta / r_{0}=0.0061$ and $\Delta z / r_{0}=0.0072$, corresponding to $\Delta r / \delta_{\theta}(0)=0.40, r_{0} \Delta \theta / \delta_{\theta}(0)=0.34$ and $\Delta z / \delta_{\theta}(0)=0.40$ when normalized by the nozzle-exit boundary-layer momentum thickness $\delta_{\theta}(0)$. In the grid used for the tripped jets, the axial and azimuthal discretizations are the same as previously, but the radial discretization changes. There are 77 points between $r=0$ and $r_{0}$, and 31 points inside the jet boundary layers, and the radial resolution is twice as high along the lip 


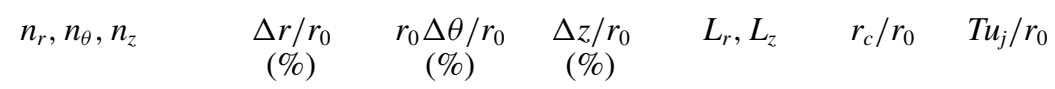

$\begin{array}{llllllll}\text { Jet0\% } & 256,1024,962 & 0.72 & 0.61 & 0.72 & 11 r_{0}, 25 r_{0} & 7.25 & 325 \\ \text { Other LESs } & 256,1024,962 & 0.36 & 0.61 & 0.72 & 9 r_{0}, 25 r_{0} & 6.5 & 375\end{array}$

TABLE 1. Simulation parameters: numbers of grid points $n_{r}, n_{\theta}, n_{z}$, mesh spacings $\Delta r$ at $r=r_{0}, r_{0} \Delta \theta$, and $\Delta z$ at $z=0$, extents $L_{r}, L_{z}$ of the physical domain, radial position $r_{c}$ of the far-field extrapolation surface, and time duration $T$.
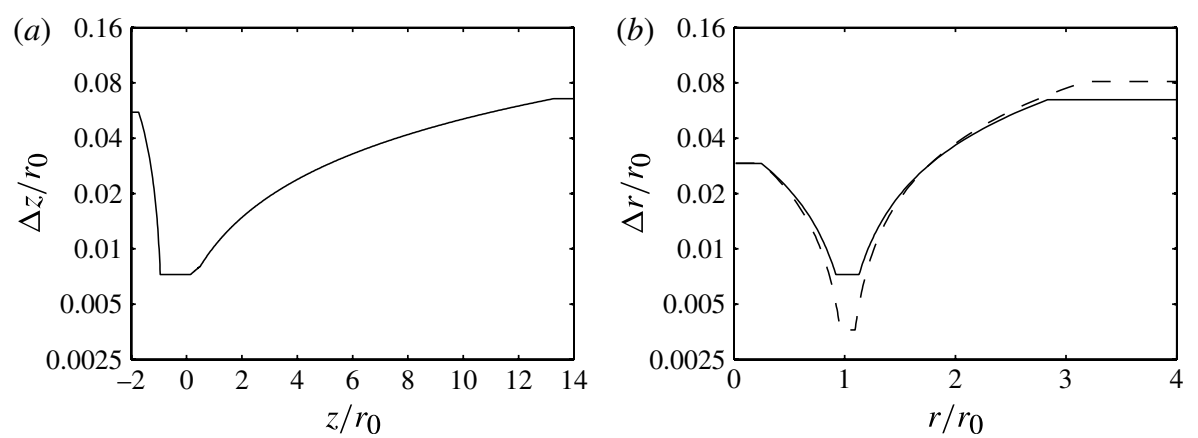

FIGURE 1. Representation, in logarithmic scales, of axial and radial mesh spacings $(a) \Delta z$ for $-2 \leqslant z / r_{0} \leqslant 14$ in all simulations and $(b) \Delta r$ for $0 \leqslant r / r_{0} \leqslant 4$ in -, Jet $0 \%$; -- , other LESs.

line with $\Delta r / r_{0}=0.0036$ at $r=r_{0}$. The physical domains, excluding the eighty-point outflow sponge zones, extend axially up to $L_{z}=25 r_{0}$, and radially up to $L_{r}=11 r_{0}$ in Jet $0 \%$ and $L_{r}=9 r_{0}$ in the other LES.

The mesh spacings are uniform in the azimuthal direction, but vary in the axial and radial directions as represented in figure 1. In figure 1(a), the axial mesh size is minimum between $z=-r_{0}$, that is the trip location in Jet3\%, Jet6\% and Jet9\%, and $z=0$, with $\Delta z / r_{0}=0.0072$. The grid is stretched upstream of $z=-r_{0}$, but also downstream of the nozzle at rates lower than $1 \%$, allowing to reach $\Delta z / r_{0}=0.065$ at $z=13.3 r_{0}$. In figure $1(b)$, the radial mesh sizes are minimum around $r=r_{0}$, with $\Delta r / r_{0}=0.0072$ in Jet $0 \%$ and $\Delta r / r_{0}=0.0036$ in the other LES. The grids are then stretched at rates lower than $4 \%$ to preserve numerical accuracy. The maximum radial mesh sizes are obtained for $r \geqslant 3 r_{0}$, and are equal to $\Delta r / r_{0}=0.065$ in Jet $0 \%$ and $\Delta r=0.081 r_{0}$ otherwise, yielding Strouhal numbers of $S t_{D}=f D / u_{j}=8.6$ and 6.9 for acoustic waves discretized by four points per wavelength ( $f$ is the time frequency).

The quality of the shear-layer discretization in Jet9\% was assessed in Bogey et al. (2011a). In that reference, the ratios between the integral length scales of the axial fluctuating velocity and the local mesh sizes along the lip line are considered. They typically fall within a range of $4-10$, suggesting that the grid resolution is appropriate in the three coordinate directions. An additional simulation is also carried out using the same grid for an axisymmetric mixing layer in which all nozzle-exit conditions are identical except for the boundary-layer thickness which is doubled. The properties of the initial turbulence and of the shear-layer flow fields obtained in Jet $9 \%$ and the latter simulation are in good agreement, showing that they are practically grid-converged. 
The solutions from Jet9\%, as well as from Jet3\%, Jet6\%, and Jet $12 \%$ using the same grid, are consequently expected to be accurate. Despite a lower radial resolution, leading to a total of 19 points within the inlet boundary layers instead of 31, the remark above most probably also applies to the results from Jet0\% because of the initially fully laminar state of the jet in that case. This assertion is supported by LES of the same kind reported in Bogey \& Bailly (2010), providing very similar results for an initially fully laminar jet using 7 or 14 points within the upstream boundary layers.

The simulation times, given in table 1 , are equal to $325 r_{0} / u_{j}$ in Jet0\% and $375 r_{0} / u_{j}$ in the other LES. Density, velocity components and pressure are recorded from time $t=125 r_{0} / u_{j}$ at every point along the jet axis, and on the two surfaces at $r=r_{0}$ and $r=r_{c}=7.25 r_{0}$ in Jet0\% $\left(t=100 r_{0} / u_{j}\right.$ and $r_{c}=6.5 r_{0}$ otherwise $)$, at a sampling frequency allowing the computation of spectra up to a Strouhal number of 20 . The velocity spectra are evaluated from overlapping samples of duration $27.4 r_{0} / u_{j}$. The flow statistics are determined from $t=175 r_{0} / u_{j}$, and they are averaged in the azimuthal direction.

The simulations have been performed using NEC SX-8 computers, on seven processors using OpenMP, leading to a CPU speed of around 36 Gflops. In particular, each of the four LES of tripped jets required around $7000 \mathrm{CPU}$ hours and $60 \mathrm{~GB}$ of memory for 164000 time steps.

\subsection{Far-field extrapolation}

The LES near fields are propagated to the acoustic far field by solving the isentropic linearized Euler equations (ILEE) in cylindrical coordinates. The extrapolation is performed from fluctuating velocities and pressure recorded in the LES on a surface at $r=r_{c}$ as reported in the previous section. These data are interpolated onto a cylindrical surface discretized by an axial mesh spacing of $\Delta z=0.065 r_{0}$. They are then imposed at the bottom boundary of the grid on which the ILEE are solved using the same numerical schemes and boundary conditions as in the LES. This grid contains $n_{r} \times n_{\theta} \times n_{z}=835 \times 256 \times 1155$ points for Jet0\%, or $845 \times 256 \times 1155$ points for the other jets, and extends axially from $z=-16.6 r_{0}$ to $58.2 r_{0}$ and radially up to $r=61.4 r_{0}$. The grid spacings are uniform with $\Delta r=\Delta z=0.065 r_{0}$, yielding a Strouhal number of $S t_{D}=8.6$ for four points per wavelength. After a propagation time of $t=60 r_{0} / u_{j}$, pressure is recorded around the jets at a distance of $60 r_{0}$ from $z=r=0$, where far-field acoustic conditions are expected to apply according to the experiments of Ahuja, Tester \& Tanna (1987), for periods of $175 r_{0} / u_{j}$ for Jet0\%, or $250 r_{0} / u_{j}$ otherwise. Pressure spectra are evaluated using overlapping samples of duration $38 r_{0} / u_{j}$, and they are averaged in the azimuthal direction.

\section{Results}

\subsection{Initial flow conditions}

\subsubsection{Mean and turbulent velocity profiles}

The profiles of mean and root-mean-square (r.m.s.) axial velocities obtained at the jet pipe exit are presented in figure 2. The mean velocity profiles, in figure 2(a), are all similar to the Blasius profile specified at the pipe inlet, leading to the exit boundary-layer momentum thicknesses and shape factors, collected in table 2, varying only from $\delta_{\theta}(0)=0.0175 r_{0}$ and $H=2.55$ in Jet $0 \%$ to $\delta_{\theta}(0)=0.0188 r_{0}$ and $H=2.33$ in Jet $12 \%$. In figure $2(b)$, the peak intensities of velocity fluctuations are found to be respectively close to $0,3 \%, 6 \%, 9 \%$ and $12 \%$, as intended; refer to table 2 for the exact values. In particular, the peak intensity is $u_{e}^{\prime} / u_{j}=0.25 \%$ in Jet $0 \%$, which 

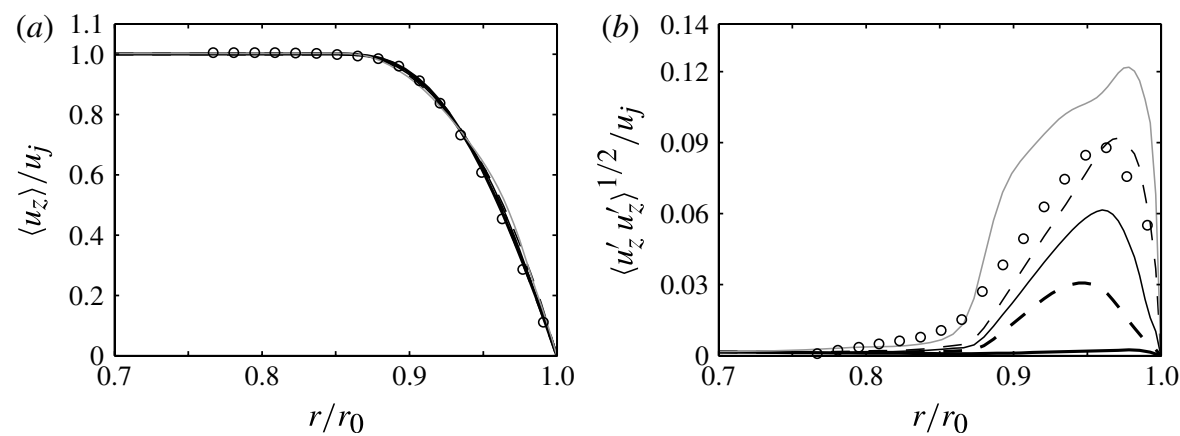

FIGURE 2. Profiles at $z=0(a)$ of mean axial velocity $\left\langle u_{z}\right\rangle$, and $(b)$ of the r.m.s. values of fluctuating axial velocity $u_{z}^{\prime}$ : - Jet0\%; - - , Jet3\%; —, Jet6\%; ---, Jet9\%; —, Jet12\%; ○, measurements of Zaman $(1985 a, b)$ for a Mach 0.18 , tripped jet at $R e_{D}=10^{5}$.

$\begin{array}{lcccccccccc} & \delta_{\theta}(0) / r_{0} & H & u_{e}^{\prime} / u_{j}(\%) & L_{u u}^{(z)} / r_{0} & L_{u u}^{(\theta)} / r_{0} & L_{u u}^{(z)} / L_{u u}^{(\theta)} & \left.a_{u u}^{(\theta)}\right|_{0} & \left.a_{u u}^{(\theta)}\right|_{1} & \left.a_{u u}^{(\theta)}\right|_{2} & \left.\sum a_{u u}^{(\theta)}\right|_{n_{\theta} \geqslant 21} \\ \text { Jet0\% } & 0.0175 & 2.55 & 0.25 & 0.450 & 2.059 & 0.22 & 0.664 & 0.283 & 0.041 & 2 \times 10^{-8} \\ \text { Jet3\% } & 0.0176 & 2.52 & 3.07 & 0.156 & 0.144 & 1.08 & 0.043 & 0.042 & 0.028 & 0.650 \\ \text { Jet6\% } & 0.0179 & 2.48 & 6.15 & 0.083 & 0.019 & 4.47 & 0.006 & 0.012 & 0.013 & 0.796 \\ \text { Jet9\% } & 0.0185 & 2.36 & 9.18 & 0.057 & 0.013 & 4.53 & 0.004 & 0.007 & 0.008 & 0.844 \\ \text { Jet12\% } & 0.0188 & 2.33 & 12.19 & 0.054 & 0.010 & 5.33 & 0.003 & 0.006 & 0.006 & 0.842\end{array}$

TABLE 2. Boundary-layer momentum thickness $\delta_{\theta}(0)$, shape factor $H$, and peak turbulence intensities $u_{e}^{\prime}$ at the nozzle exit, and flow properties calculated from velocity $u_{z}^{\prime}$ at $r=r_{0}$ and $z=0.4 r_{0}$ : axial and azimuthal integral length scales $L_{u u}^{(z)}$ and $L_{u u}^{(\theta)}$, ratio $L_{u u}^{(z)} / L_{u u}^{(\theta)}$, and coefficients $\left.a_{u u}^{(\theta)}\right|_{n_{\theta}}$ obtained from the cross-correlation functions $\mathscr{R}_{u u}^{(\theta)}$.

indicates a feedback mechanism in this jet simulation without boundary-layer trip-like forcing, as mentioned in $\S 2.1$. According to Zaman $(1985 a, b)$, the present jets can be considered to be in the following initial states: fully laminar in Jet0\%, nominally laminar in Jet3\%, and nominally turbulent (or highly disturbed) in Jet6\%, Jet9\% and Jet $12 \%$. The initial conditions in Jet $9 \%$ are also shown to be comparable to those measured in a tripped jet at $\operatorname{Re}_{D}=10^{5}$ by the above author.

\subsubsection{Length-scale and azimuthal distributions}

Given their importance in characterizing the inflow, the integral length scales $L_{u u}^{(z)}$ and $L_{u u}^{(\theta)}$ estimated from the axial fluctuating velocity $u_{z}^{\prime}$ in the axial and azimuthal directions at $r=r_{0}$ and $z=0.4 r_{0}$ are presented in figure $3(a)$ and in table 2. They both decrease monotonically with $u_{e}^{\prime} / u_{j}$, from $L_{u u}^{(z)}=0.45 r_{0}$ down to $L_{u u}^{(z)}=0.054 r_{0}$, and from $L_{u u}^{(\theta)}=2.1 r_{0}$ down to $L_{u u}^{(\theta)}=0.010 r_{0}$. The reduction is quite sharp for the azimuthal length scales, as previously noted in Bogey \& Bailly (2010). In particular, the azimuthal correlation of disturbances just downstream of the pipe lip is very high in the untripped jet, but is quite low in Jet6\%, Jet9\% and Jet12\% with integral length scales roughly equal to or slightly smaller than the boundary-layer momentum thickness $\delta_{\theta}(0)$ in these jets.

The nature of initial turbulence in the shear layers therefore appears to change fundamentally with the nozzle-exit fluctuation level. To demonstrate this, the values of 

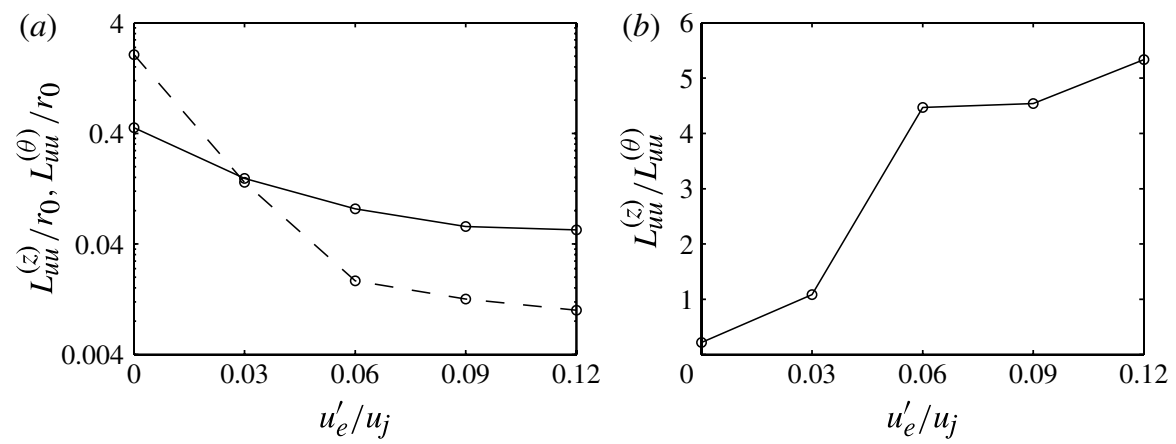

FIGURE 3. Variations with the exit turbulence level $u_{e}^{\prime} / u_{j}:(a)$ of the axial and azimuthal integral length scales,$- L_{u u}^{(z)} ;---, L_{u u}^{(\theta)}$; and $(b)$ the ratio $L_{u u}^{(z)} / L_{u u}^{(\theta)}$, calculated from velocity $u_{z}^{\prime}$ at $r=r_{0}$ and $z=0.4 r_{0}$.

the ratio $L_{u u}^{(z)} / L_{u u}^{(\theta)}$ between integral length scales at $r=r_{0}$ and $z=0.4 r_{0}$ are displayed in figure $3(b)$ and given in table 2. It turns out that $L_{u u}^{(z)} / L_{u u}^{(\theta)}=0.22$ in Jet0\% whereas $L_{u u}^{(z)} / L_{u u}^{(\theta)} \simeq 5$ for $u_{e}^{\prime} / u_{j} \geqslant 6 \%$. The structures initially dominating in the mixing layers are consequently mainly elongated in the azimuthal direction in the untripped jet, but in the axial direction in the initially highly disturbed jets. In the latter case they correspond most likely to the structures observed in turbulent boundary layers, described for instance in the experiments of Tomkins \& Adrian (2003, 2005), as will be discussed below.

In order to quantify the modal distribution of the jet initial disturbances, the Fourier coefficients $\left.a_{u u}^{(\theta)}\right|_{n_{\theta}}$ obtained from the decomposition of cross-correlation functions $\mathscr{R}_{u u}^{(\theta)}$ of velocity $u_{z}^{\prime}$ with the azimuthal modes $n_{\theta}$ are calculated at $r=r_{0}$ and $z=0.4 r_{0}$, as was done previously in Bogey \& Bailly (2010). The relative amplitudes of the first modes $n_{\theta}=0,1$ and 2 are represented in figure 4 and provided in table 2 . As expected, the contributions of these modes are predominant in Jet0\%, but weaken appreciably as the nozzle-exit turbulence intensity increases. The total contribution of modes $n_{\theta} \geqslant 21$ corresponding to azimuthal wavelengths $\lambda_{\theta} \leqslant 2 \delta$, smaller than twice the boundary-layer thickness, is also reported in figure 4 and in table 2 . Contrary to the contributions of the first modes, this contribution is negligible in the untripped jet, but is higher than $60 \%$ in the tripped jets. This result further supports that initial turbulence in these jets corresponds to typical boundary-layer turbulence.

\subsubsection{Velocity spectra}

The properties of the jet initial disturbances are examined in more detail by calculating spectra of the fluctuating axial velocity at the same position as previously. The spectra are represented as a function of the Strouhal number $S t_{D}=f D / u_{j}$ in figure $5(a)$, and of the azimuthal mode $n_{\theta}$ in figure $5(b)$. Those obtained for the untripped jet show typical features of an initially fully laminar jet. The initial turbulence, albeit at a low level, is indeed dominated in Jet $0 \%$ by highly distinctive frequency and azimuthal components. In particular, the contributions of the first azimuthal modes appear very high, whereas those from modes $n_{\theta}>2$ are negligible, as was quantified above.

The spectra obtained in the tripped jets all display broadband shapes, which are seen not to change much with the initial fluctuation level. For these jets, the frequency spectra in figure $5(a)$ are rather flat up to $S t_{D} \simeq 1$ and rapidly decrease 


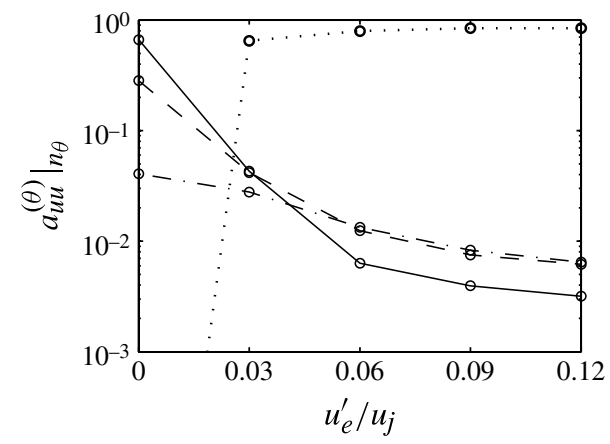

FIGURE 4. Variations with $u_{e}^{\prime} / u_{j}$ of the coefficients $\left.a_{u u}^{(\theta)}\right|_{n_{\theta}}$ obtained from the cross-correlation functions $\mathscr{R}_{u u}^{(\theta)}$ of velocity $u_{z}^{\prime}$ at $r=r_{0}$ and $z=0.4 r_{0}:-, n_{\theta}=0 ;---, n_{\theta}=1 ;-\cdot-\cdot, n_{\theta}=2$; $\cdots \cdots$, , sum for $n_{\theta} \geqslant 21$.
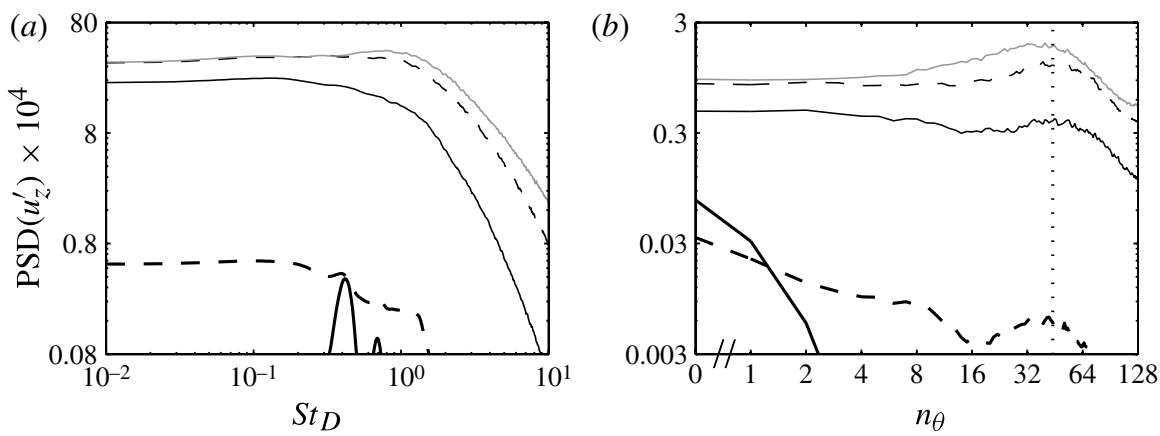

FIGURE 5. Power spectral densities (PSD) normalized by $u_{j}$ of fluctuating velocity $u_{z}^{\prime}$ at $r=r_{0}$ and $z=0.4 r_{0}$, as functions $(a)$ of Strouhal number $S t_{D}=f D / u_{j}$ and $(b)$ of azimuthal mode $n_{\theta}$ : - Jet0\%; ---, Jet3\%; - Jet6\%; ---, Jet $9 \%$; - Jet $12 \%$. The dotted line indicates $n_{\theta}=44$.

for higher Strouhal numbers, while the spectra in figure $5(b)$ show a distribution of turbulent energy over a wide range of azimuthal modes. In the latter spectra moreover, significant components clearly emerge around $n_{\theta} \simeq 44$. These components are weaker than the first low-order modes $n_{\theta}=0,1,2$ in Jet3\%. However, as their relative contributions increase with the nozzle-exit turbulence intensity, they are dominating in Jet9\% and Jet $12 \%$ for the two jets with highest initial disturbances.

The physical relevance of the initial velocity spectra in Jet $9 \%$ was recently discussed in Bogey et al. (2011b), by re-plotting them versus axial and azimuthal wavenumbers $k_{z} \delta$ and $k_{\theta} \delta \quad\left(k_{\theta}=n_{\theta} / r_{0}\right)$, using a scaling with the boundary-layer thickness $\delta$ frequently encountered for wall-bounded flows. The same normalization is applied for the present spectra in figure 6. Spectra obtained by Eggels et al. (1994) in a fully turbulent pipe flow using direct numerical simulation (DNS) are also represented for comparison. A good qualitative agreement is found between the shapes of the DNS spectra and those of the LES spectra for $u_{e}^{\prime} / u_{j} \geqslant 6 \%$. In particular, the wavenumbers emerging in the azimuthal direction match relatively well. The peak wavenumber value $k_{\theta} \delta=6.6$ associated with $n_{\theta}=44$ is also consistent with measurements of spanwise energy distribution in turbulent boundary layers provided by Tomkins \& Adrian (2005). 

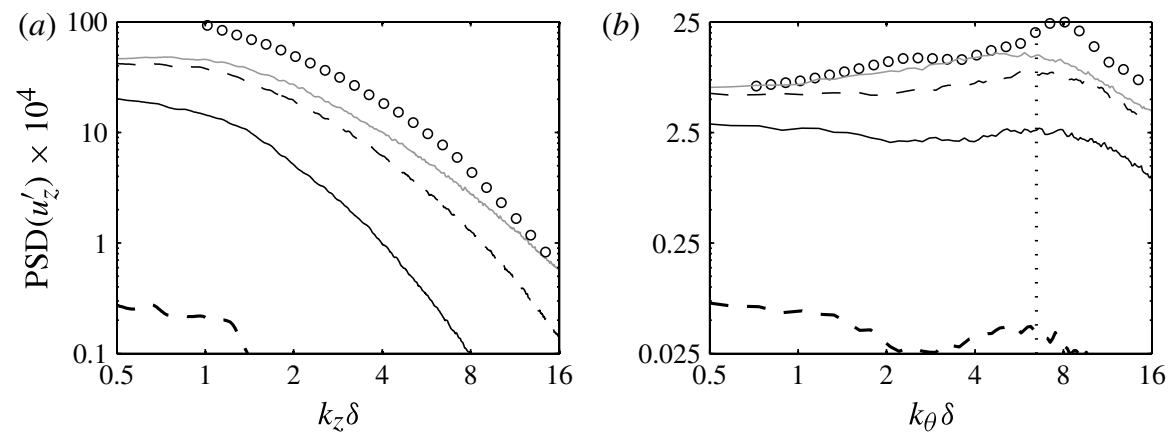

FIgURE 6. Power spectral densities (PSD) normalized by $u_{j}$ of velocity $u_{z}^{\prime}$ at $r=r_{0}$ and $z=0.4 r_{0}$, as functions of non-dimensional axial and azimuthal wavenumbers $(a) k_{z} \delta$ and (b) $k_{\theta} \delta$ : ---, Jet3\%; - Jet6\%; ---, Jet9\%; —, Jet12\%; o, DNS data of Eggels et al. (1994) for a fully turbulent pipe flow, shifted arbitrarily in magnitude for the comparison. The dotted line indicates $k_{\theta} \delta=6.6$.

This can be expected given the similarities between turbulent pipe flows and boundary layers reported by Monty et al. (2009) and Ghosh, Foysi \& Friedrich (2010), among others. The initial turbulent structures in the tripped jets are therefore organized in a similar fashion to those in turbulent wall-bounded flows.

\subsection{Shear-layer development}

\subsubsection{Vorticity fields}

Vorticity fields obtained downstream of the pipe lip are represented up to $z=3.75 r_{0}$ in figure 7 and supplementary movie 1 available at journals.cambridge.org/flm, and up to $z=10 r_{0}$ in supplementary movie 2. For low initial velocity fluctuations in $\mathrm{Jet} 0 \%$ and Jet $3 \%$, the shear layers are (fully or nominally) laminar at $z=0$, leading to laminar-turbulent flow transitions dominated by roll-ups and pairings of large vortical structures. With increasing nozzle-exit disturbance level, the shear layers tend towards being initially turbulent, which naturally makes vortex roll-up disappear. More interestingly, the development of the mixing layers gradually displays enhanced finescale turbulence as well as weaker large-scale structures. This is especially the case for Jet $12 \%$ in figure $7(e)$, where it is difficult to distinguish large-scale structures or pairing processes in the shear layer.

In order to shed light on the generation of three-dimensional motions in the mixing layers, snapshots of the axial vorticity $\omega_{z}$ obtained in three sections located at $z=r_{0}$, $z=2 r_{0}$ and $z=4 r_{0}$ are presented in figure 8 . In Jet0\%, in figure $8(a)$, the axial vorticity field is of negligible amplitude at $z=0$, shows a small number of spots of significant level at $z=2 r_{0}$, and then is developed at $z=4 r_{0}$. This indicates that the flow in the initially fully laminar jet is nearly axisymmetric at the first position, starts its transition towards three-dimensionality around the second one, and is turbulent at the third one, in agreement with the vorticity snapshot of figure $7(a)$. In Jet3\%, threedimensional structures are already clearly visible at $z=2 r_{0}$ in figure $8(b)$, implying that the laminar-turbulent flow transition occurs farther upstream in that case, as can also be inferred from figure $7(b)$. In the three other LES dealing with initially highly disturbed jets, unsurprisingly, three-dimensional turbulent fields are observed in figure $8(c-e)$ in the three planes considered. These fields may however contain more fine scales for higher initial turbulence levels. 

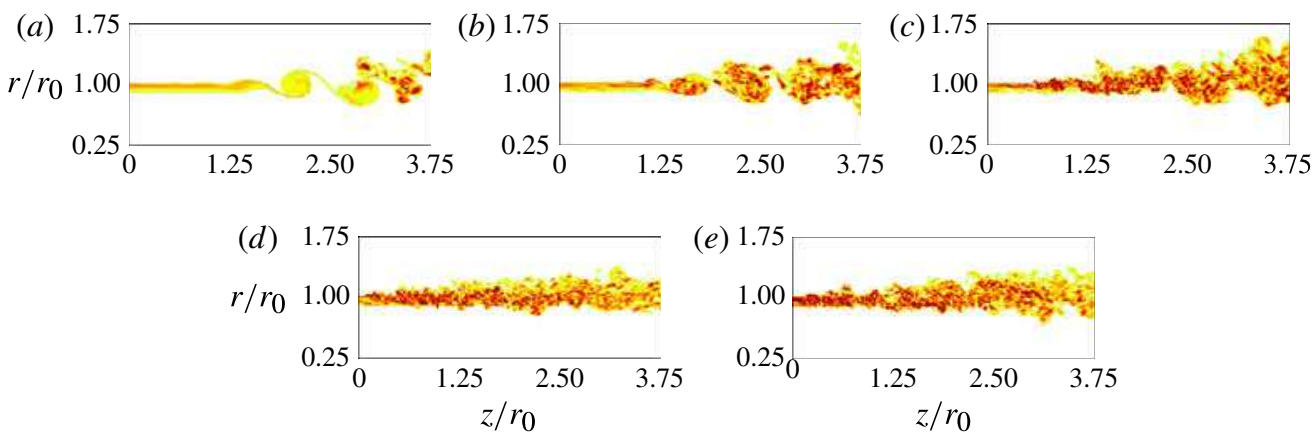

FIGURE 7. (Colour online available at journals.cambridge.org/flm) Snapshots in the $(z, r)$ plane of vorticity norm $|\omega|$ in the shear layer just downstream of the pipe lip: $(a)$ Jet0\%, (b) Jet3\%, (c) Jet6\%, (d) Jet9\%, (e) Jet12\%. The colour scale ranges up to the level of $25 u_{j} / r_{0}$.

\subsubsection{Length scales and mean and turbulent velocity profiles}

To obtain rough approximations of the sizes of the turbulent structures in the mixing layers, the axial and azimuthal integral length scales $L_{u u}^{(z)}$ and $L_{u u}^{(\theta)}$ are evaluated from velocity $u_{z}^{\prime}$ at $r=r_{0}$. Their variations between $z=0$ and $z=10 r_{0}$ are presented in figure 9 . In figure $9(a)$, after a transitional period corresponding very probably to the flow adjustment following the nozzle lip from a boundary-layer profile to a shear-layer profile, the axial length scales are observed to grow fairly linearly, which is in agreement with the experimental data available for jets in Davies, Fisher \& Barratt (1963) and in Fleury et al. (2008) for instance. These scales are seen to become gradually smaller as the jet initial turbulence intensity increases. Similar trends are noted for the azimuthal length scales in figure $9(b)$, as expected from measurements of spanwise velocity correlations in plane mixing layers by Jones, Planchon \& Hammersley (1973) and Browand \& Troutt (1980). The influence of the exit disturbance level, however, appears stronger on the azimuthal than on the axial length scales, especially during the early stage of mixing-layer development. Downstream of the nozzle, the azimuthal length scales are indeed much larger in Jet0\% and Jet3\% than in the other LES.

The variations over $0 \leqslant z \leqslant 10 r_{0}$ of the shear-layer momentum thickness $\delta_{\theta}$ are presented in figure $10(a)$. With increasing nozzle-exit turbulence level, as expected from the vorticity fields of figure 7 and supplementary movies 1 and 2 , the mixinglayer growth begins closer to the exit section, at a position ranging from $z \simeq 1.5 r_{0}$ in Jet $0 \%$ to $z \simeq 0$ in Jet9\% and Jet $12 \%$. It then occurs at a much lower rate resulting in a slower overall flow development. To quantify this point, the variations of the spreading rate $\mathrm{d} \delta_{\theta} / \mathrm{d} z$ are shown in figure $10(b)$. Higher initial disturbances in the jets clearly lead to lower spreading rates. As further illustrated in figure 12(a) below, the decrease is significant for their mean values from the nozzle exit to the end of the jet potential core, as well as for their peak values, which are equal to 0.057 in Jet0\%, 0.045 in Jet3\%, 0.033 in Jet6\% and 0.024 in Jet9\%. Similar behaviour has been found experimentally for mixing layers by Hussain \& Zedan (1978b), Browand \& Latigo (1979), Husain \& Hussain (1979), and Bell \& Mehta (1990). Husain \& Hussain (1979) notably considered axisymmetric mixing layers with laminar or turbulent upstream conditions, characterized by Reynolds numbers $R e_{\theta} \simeq 400$ and 

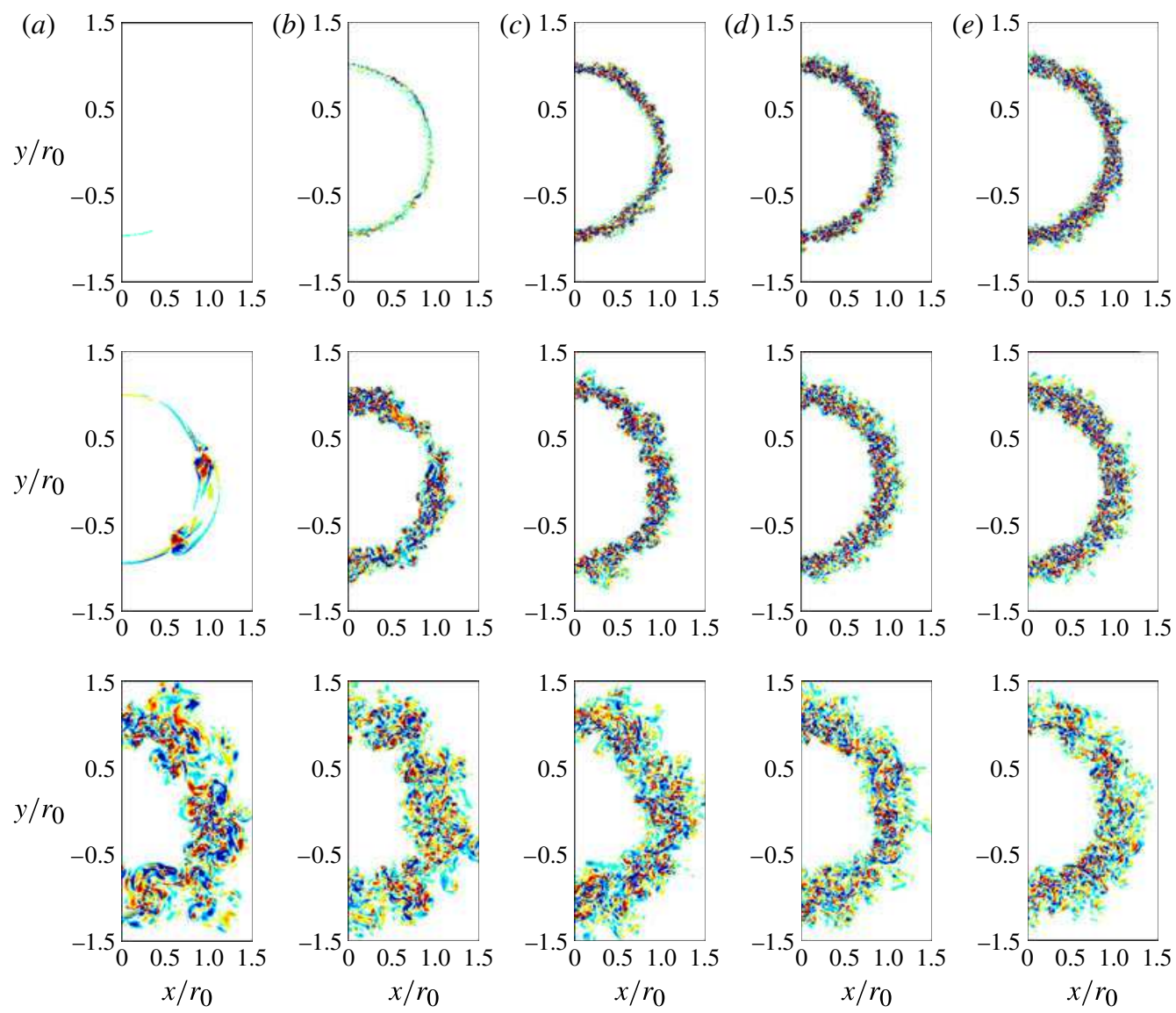

FIGURE 8. (Colour online) Snapshots in the $(r, \theta)$ or $(x, y)$ planes at $z=r_{0}, 2 r_{0}$ and $4 r_{0}$, from top to bottom, of axial vorticity $\omega_{z}:(a) \mathrm{Jet} 0 \%$, (b) Jet3\%, (c) Jet6\%, (d) Jet9\%, (e) Jet12\%. The colour scale ranges from $-13 u_{j} / r_{0}$ to $13 u_{j} / r_{0}$. Only the half-plane $x \geqslant 0$ is shown.
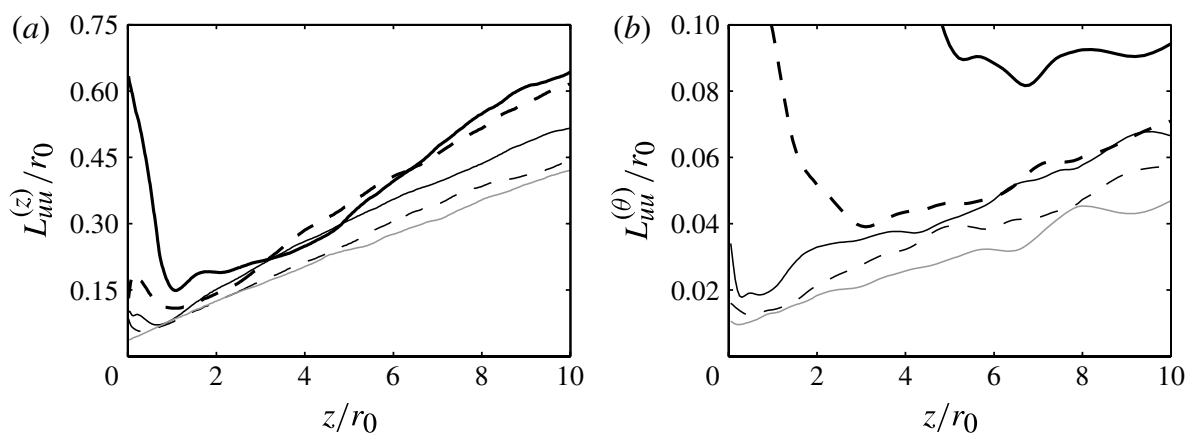

FIGURE 9. Variations of the axial and azimuthal integral length scales $(a) L_{u u}^{(z)}$ and $(b) L_{u u}^{(\theta)}$ calculated from velocity $u_{z}^{\prime}$ at $r=r_{0}$ : - Jet0\%; ---, Jet3\%; - Jet6\%; ---, Jet9\%; Jet $12 \%$. 

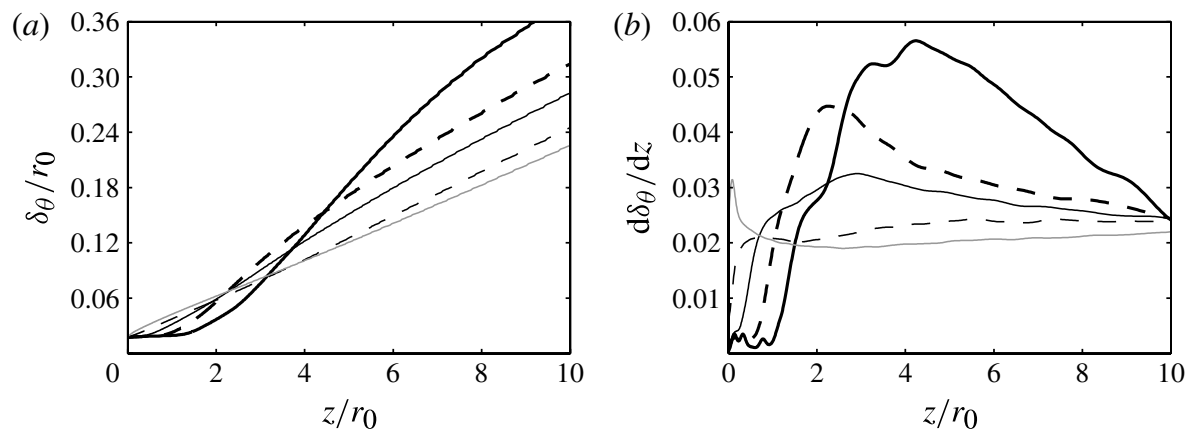

FIGURE 10. Variations ( $a$ ) of shear-layer momentum thickness $\delta_{\theta}$ and $(b)$ of spreading rate $\mathrm{d} \delta_{\theta} / \mathrm{d} z:-$, Jet0\%; -- - Jet3\%; —, Jet6\%;---, Jet9\%; —, Jet12\%.

initial turbulence intensities $u_{e}^{\prime} / u_{j} \simeq 3 \%$ in the former case, and by $R e_{\theta} \simeq 1400$ and $u_{e}^{\prime} / u_{j} \simeq 10 \%$ in the latter. They obtained maximum spreading rates around 0.07 for laminar conditions but around 0.03 for turbulent conditions, which agrees fairly well with the present results.

The peak r.m.s. values of axial, radial and azimuthal velocities $u_{z}^{\prime}, u_{r}^{\prime}$ and $u_{\theta}^{\prime}$ and the maximum Reynolds shear stresses $\left\langle u_{r}^{\prime} u_{z}^{\prime}\right\rangle$ are represented between $z=0$ and $z=10 r_{0}$ in figure 11 . In Jet $0 \%$, maximum intensities around $22 \%$ are achieved for all velocity components. The profiles also exhibit dual-peak shapes, which is typical of a first stage of strong vortex pairings in the mixing layers according to experimental and numerical data from Zaman \& Hussain (1980) and Bogey \& Bailly (2010), among others. As $u_{e}^{\prime} / u_{j}$ increases, the turbulence intensities start to rise farther upstream in the shear layers, but lower peak values are reached. The reduction in r.m.s. velocities and Reynolds shear stresses is significant, as shown in table 3 and in figure $12(b)$. This is especially the case for the radial velocity, whose maximum r.m.s. values with respect to the jet velocity range from $22.6 \%$ in Jet0\% down to $10.7 \%$ in Jet $12 \%$, while they are $17.7 \%$ in Jet $3 \%, 13.7 \%$ in Jet6\% and $11.2 \%$ in Jet $9 \%$. The turbulence intensity profiles finally increase nearly monotonically in Jet9\% and Jet $12 \%$, toward values $\left\langle u_{z}^{\prime 2}\right\rangle^{1 / 2} / u_{j} \simeq 15 \%$ and $\left\langle u_{r}^{\prime 2}\right\rangle^{1 / 2} / u_{j} \simeq 11 \%$ for example. In Jet12\%, in particular, the profiles become strikingly flat quickly downstream of the nozzle exit. These results correspond nicely to those obtained experimentally in plane and axisymmetric mixing layers by Browand \& Latigo (1979) and Husain \& Hussain (1979). The profiles of axial turbulence intensity were indeed found rapidly to reach a peak of around $20 \%$ for laminar initial conditions with $u_{e}^{\prime} / u_{j} \simeq 3 \%$, but to relax monotonically to an asymptotic value around $16 \%$ for turbulent initial conditions with $u_{e}^{\prime} / u_{j} \simeq 10 \%$. Monotonic variations can similarly be noted for the turbulence intensities measured by Arakeri et al. (2003) in a jet at $R e_{D}=5 \times 10^{5}$ with $u_{e}^{\prime} / u_{j} \simeq 10 \%$, the r.m.s. axial velocities tending to $\sim 14 \%$ of the jet velocity in that case.

The present results, summarized in figure 12, show that the development of the mixing layers becomes smoother with rising nozzle-exit turbulence intensity. The peak r.m.s. values of velocity obtained for turbulent exit conditions, namely for $u_{e}^{\prime} / u_{j} \simeq 10 \%$, are roughly half those found for fully laminar exit conditions. This trend can reasonably be attributed to the strong weakening of large-scale vortices and of their interactions in the latter case, which will be examined in what follows based on an in-depth analysis of the shear-layer turbulence. 

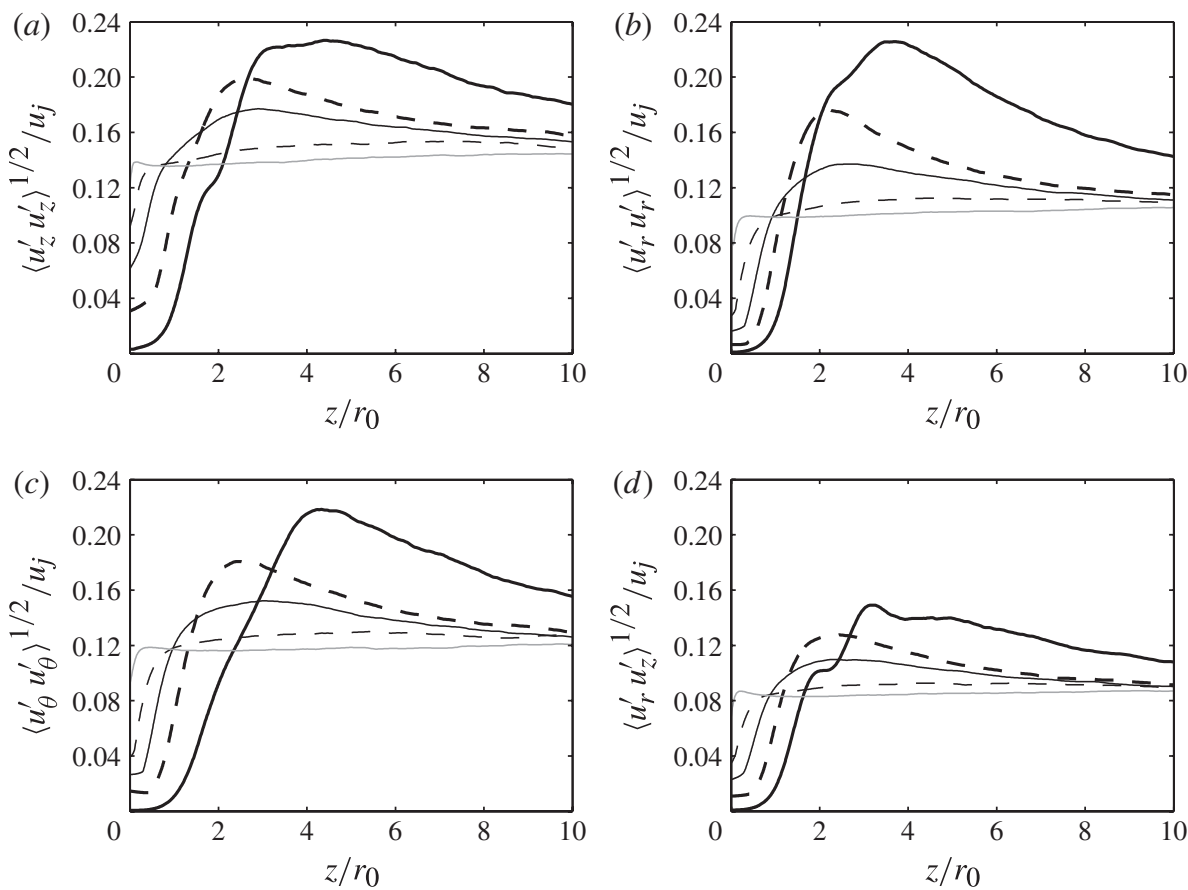

FIGURE 11. Variations of the peak r.m.s. values of fluctuating velocities (a) $u_{z}^{\prime}$, (b) $u_{r}^{\prime}$, (c) $u_{\theta}^{\prime}$, and $(d)$ of the peak magnitudes of Reynolds shear stress $\left\langle u_{r}^{\prime} u_{z}^{\prime}\right\rangle:-$, Jet0\%; ---, Jet3\%; Jet6\%;---, Jet9\%; - Jet12\%.

$\begin{array}{lccc} & \left\langle u_{z}^{\prime 2}\right\rangle^{1 / 2} / u_{j}(\%) & \left\langle u_{r}^{\prime 2}\right\rangle^{1 / 2} / u_{j}(\%) & \left\langle u_{\theta}^{\prime 2}\right\rangle^{1 / 2} / u_{j}(\%) \\ \text { Jet0\% } & 22.7 & 22.6 & 21.8 \\ \text { Jet3\% } & 19.9 & 17.6 & 18.1 \\ \text { Jet6\% } & 17.7 & 13.7 & 15.2 \\ \text { Jet9\% } & 15.4 & 11.2 & 13 \\ \text { Jet12\% } & 14.5 & 10.6 & 12.2\end{array}$

TABLE 3. Peak r.m.s. values of fluctuating velocities $u_{z}^{\prime}, u_{r}^{\prime}$ and $u_{\theta}^{\prime}$ in the jets.

\subsubsection{Structure of turbulent fields}

To check the three-dimensionality of the turbulent fields in the mixing layers, the variations of the ratio $\left\langle u_{\theta}^{\prime} u_{\theta}^{\prime}\right\rangle /\left(\left\langle u_{z}^{\prime} u_{z}^{\prime}\right\rangle+\left\langle u_{r}^{\prime} u_{r}^{\prime}\right\rangle+\left\langle u_{\theta}^{\prime} u_{\theta}^{\prime}\right\rangle\right)$ at $r=r_{0}$ are plotted in figure $13(a)$. In Jet0\%, this ratio is typically lower than 0.1 between $z=0$ and $z \simeq 1.5 r_{0}$, indicating the presence of strong two-dimensional structures in the early stage of mixing-layer transition. It grows farther downstream as two-dimensionality gradually disappears, and reaches values around 0.3 for $z \geqslant 4 r_{0}$, where turbulence can then be regarded as fully three-dimensional. Similar behaviours are observed for the tripped jets. However, as the level of the jet initial disturbances increases, azimuthal velocity contributes more significantly to the turbulent kinetic energy close to the nozzle exit, and values around $30 \%$ are achieved more rapidly. This is particularly 

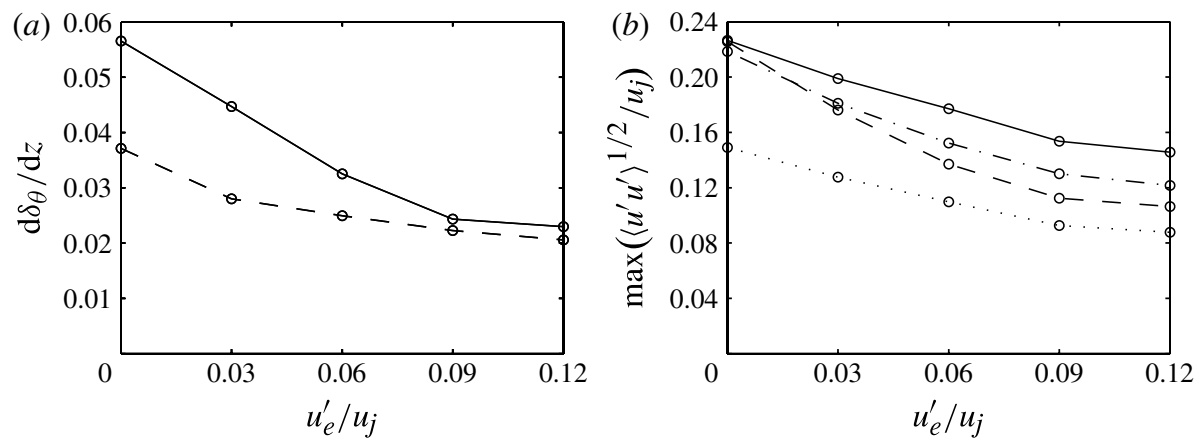

FIGURE 12. Variations with the exit turbulence level $u_{e}^{\prime} / u_{j},(a)$ of the shear-layer spreading rate $\mathrm{d} \delta_{\theta} / \mathrm{d} z$, - , peak values between $z=r_{0}$ and $z_{c} ;---$, mean values between $z=0$ and $z_{c}$; and $(b)$ of the peak values of turbulence intensities - $-\left\langle u_{z}^{\prime 2}\right\rangle^{1 / 2} / u_{j} ;---,\left\langle u_{r}^{\prime 2}\right\rangle^{1 / 2} / u_{j} ;-\cdot-\cdot$, $\left\langle u_{\theta}^{\prime 2}\right\rangle^{1 / 2} / u_{j} ; \ldots \ldots,\left\langle u_{r}^{\prime} u_{z}^{\prime}\right\rangle^{1 / 2} / u_{j}$.
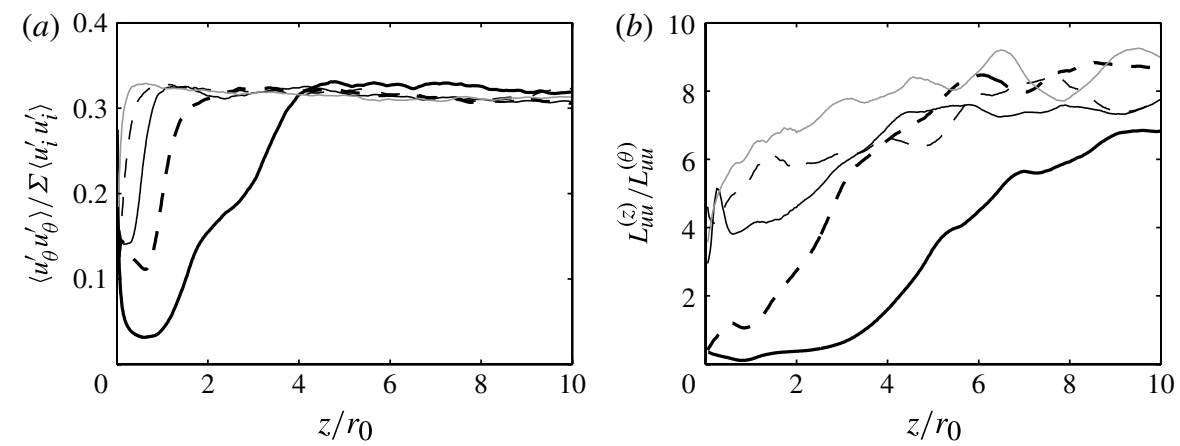

FIGURE 13. Variations $(a)$ of the ratio $\left\langle u_{\theta}^{\prime} u_{\theta}^{\prime}\right\rangle /\left(\left\langle u_{z}^{\prime} u_{z}^{\prime}\right\rangle+\left\langle u_{r}^{\prime} u_{r}^{\prime}\right\rangle+\left\langle u_{\theta}^{\prime} u_{\theta}^{\prime}\right\rangle\right)$, and $(b)$ of the ratio $L_{u u}^{(z)} / L_{u u}^{(\theta)}$ between axial and azimuthal integral length scales, at $r=r_{0}$ : - Jet0\%; - - , Jet3\%; - Jet6\%; ---, Jet9\%; - , Jet12\%.

the case in Jet9\% and Jet12\%, in which three-dimensional features are found to predominate quickly downstream of the nozzle.

Another way to evaluate the persistence of two-dimensional structures in plane or axisymmetric mixing layers, followed by Chandrsuda et al. (1978) for example, consists of considering the changes in velocity correlations in the spanwise or azimuthal directions. For the present jets, the ratios $L_{u u}^{(z)} / L_{u u}^{(\theta)}$ computed between axial and azimuthal length scales at $r=r_{0}$ are presented in figure 13(b). The profiles thus obtained show a great resemblance with those in figure $13(a)$, since the values of $L_{u u}^{(z)} / L_{u u}^{(\theta)}$ generally grow with $u_{e}^{\prime} / u_{j}$ and with increasing axial position. Moreover, they seem not to vary much, taking values close to $L_{u u}^{(z)} / L_{u u}^{(\theta)}=8$, sufficiently far from the nozzle exit, when the mixing layers are fully developed. This value compares well with experimental results in the literature. On the basis of the data of Fleury et al. (2008), the axial integral length scales in the shear layers of round jets are indeed roughly of $0.06 z$, whereas lateral length scales of $0.01 z$ were found by Jones et al. (1973) in a plane mixing layer. A ratio of $\sim 8$ between the axial and azimuthal length 

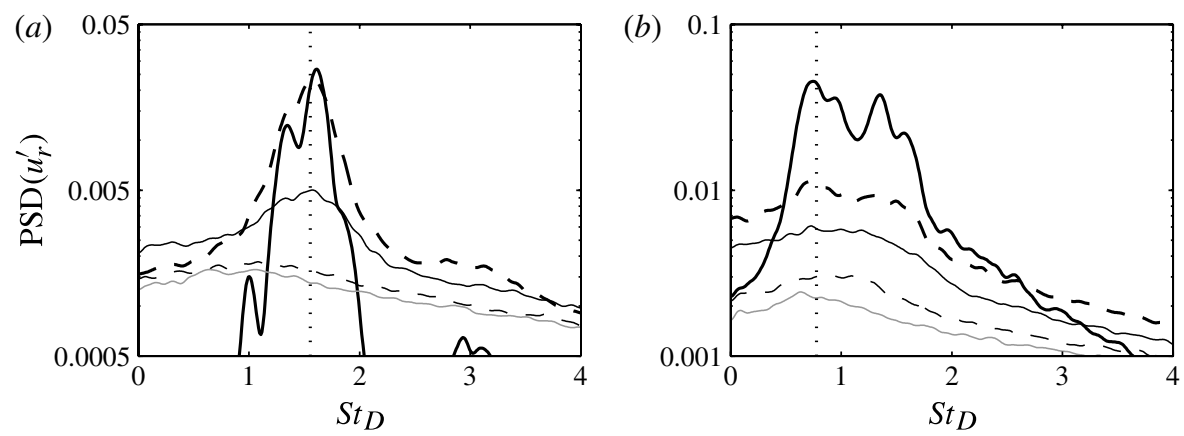

FIGURE 14. Power spectral densities (PSD) normalized by $u_{j}$ of radial velocity $u_{r}^{\prime},(a)$ at $r=r_{0}$ and $z=1.5 r_{0}$ and $(b)$ at $r=r_{0}$ and $z=3 r_{0}$, as functions of $S t_{D}=f D / u_{j}$ : -, Jet0\%; ---, Jet3\%; - Jet6\%; ---, Jet9\%; - Jet12\%. The dotted lines indicate (a) $S t_{\theta}=f \delta_{\theta}(0) / u_{j}=0.014$ and $(b) S t_{\theta}=0.007$.

scales can also be deduced from the measurements by Morris \& Zaman (2009) at $r=r_{0}$ and $z=10 r_{0}$ in a jet at $\operatorname{Re}_{D}=3 \times 10^{5}$.

Spectra of the radial velocity $u_{r}^{\prime}$ are computed at $z=1.5 r_{0}$ and $z=3 r_{0}$ at $r=r_{0}$, and plotted in figures 14(a) and 14(b), respectively, as a function of the Strouhal number $S t_{D}$. As the initial turbulence level increases, they become broader, but some similarities can be seen. For Jet $0 \%$, Jet $3 \%$ and Jet6\%, the velocity spectra at $z=1.5 r_{0}$ exhibit pronounced peak frequencies. These frequencies correspond approximately to $S t_{D}=1.6$, or to $S t_{\theta}=f \delta_{\theta}(0) / u_{j}=0.014$ when normalized by the exit boundary-layer thickness, which is typical of frequencies predominant early on in annular mixing layers according to Gutmark \& Ho (1983); refer also to the linear stability analyses conducted by Morris $(1976,1983)$ and Michalke (1984) and to that in the Appendix. At $z=3 r_{0}$, the spectra are dominated by components centred around $S t_{D}=0.8$ or $S t_{\theta}=0.007$, which is the first sub-harmonic of the peak frequency at $z=1.5 r_{0}$. These results suggest the occurrence of roll-ups and pairings of Kelvin-Helmholtz-like vortices in Jet0\%, as for the initially fully laminar jets in Bogey \& Bailly (2010), as well as in Jet3\% and Jet6\% for the two initially moderately disturbed jets. For Jet $9 \%$ and Jet $12 \%$, however, the velocity spectra display much flatter shapes, and the presence and pairings of large-scale vortices in the mixing layers seem doubtful.

To more accurately identify the mechanisms taking place in the vicinity the nozzle exit, the levels obtained in the spectra of velocity $u_{r}^{\prime}$ at $r=r_{0}$ at the Strouhal number $S t_{D}=1.6$ dominating at $z=1.5 r_{0}$ are presented in figure $15(a)$. In Jet $0 \%$, Jet $3 \%$ and Jet6\%, they clearly increase exponentially just downstream of $z=0$, implying the growth of linear instability waves early in the shear layers. The amplification rates are approximatively equal to $\alpha r_{0}=4.4$, or $\alpha \delta_{\theta}(0) \simeq 0.08$, which agrees well with the values estimated using a linear stability analysis in the Appendix for the modes $n_{\theta}=0,1$ and 2 around $S t_{\theta}=0.14$ for a base-flow profile representative of the initial mixing-layer profiles. On the contrary, the presence of linear instability waves appears unlikely in Jet9\% and Jet $12 \%$ from figure 15(a). It can also be emphasized that instability waves are generally difficult to detect in shear layers containing intense broadband turbulent fluctuations, as was pointed out by Suzuki \& Colonius (2007).

As a natural complement to figure 15(a), the peak Strouhal numbers in the spectra of $u_{r}^{\prime}$ at $r=r_{0}$ are represented in figure $15(b)$ to track the components dominating along the shear layers. The component around $S t_{D}=1.6$, associated above with linear 

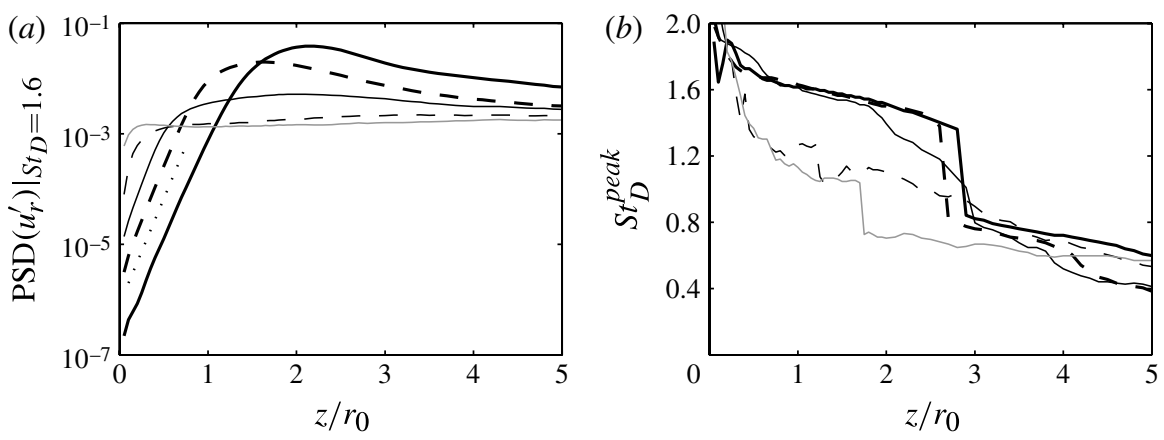

FIGURE 15. Variations $(a)$ of the level obtained at Strouhal number $S t_{D}=1.6$ and $(b)$ of the peak Strouhal number $S t_{D}^{\text {peak }}$ in the power spectral densities (PSD) normalized by $u_{j}$ of radial velocity $u_{r}^{\prime}$, at $r=r_{0}$ : - Jet0\%; ---, Jet3\%; - Jet6\%; ---, Jet9\%; - , Jet $12 \%$. The dotted line indicates a disturbance amplification rate of $\alpha r_{0}=4.4$, yielding $\alpha \delta_{\theta}(0) \simeq 0.08$.

instability waves, visibly persists in Jet0\% and Jet3\% over a wide axial extent, nearly up to $z=3 r_{0}$. A second step at $S t_{D} \simeq 0.8$ is then observed farther downstream. Similar features are found in Jet6\%, though the jump between $S t_{D}=1.6$ and $S t_{D}=0.8$ is smoother. A first stage of vortex pairings, strong in Jet $0 \%$ and Jet3\% and significantly weaker in Jet6\%, therefore takes place in the shear layers of these jets. Unfortunately, it is not possible to distinguish such turbulent events in the two initially highly disturbed jets, since frequencies around $S t_{D}=1.6$ or $S t_{D}=0.8$ do not emerge in this case. They may however still exist, albeit quite considerably attenuated, in Jet $9 \%$, notably because of the moderate Reynolds number $R e_{\theta}=900$, see the discussion in Bogey et al. (2011a) as well as recent results in Bogey et al. (2012).

As for the nozzle-exit disturbances in $\$ 3.1 .2$, the modal distribution of shear-layer turbulence in the azimuthal direction is characterized by the Fourier coefficients $\left.a_{u u}^{(\theta)}\right|_{n_{\theta}}$ of the cross-correlation functions $\mathscr{R}_{u u}^{(\theta)}$ of velocity $u_{z}^{\prime}$ at $r=r_{0}$. The contributions of modes $n_{\theta}=0,1$ and 2 , and that of modes $n_{\theta} \geqslant 21$ corresponding to wavelengths $\lambda_{\theta} \leqslant 2 \delta$, are shown in figure 16 up to $z=5 r_{0}$ for Jet $0 \%$, Jet $3 \%$, Jet $6 \%$ and Jet9\%. In $\mathrm{Jet} 0 \%$, in agreement with previous simulations of initially fully laminar jets at $R e_{D}=10^{5}$ by Kim \& Choi (2009) and Bogey \& Bailly (2010), the development of the mixing layers just downstream of the nozzle lip is governed by the first azimuthal modes. The relative amplitude of the axisymmetric mode initially is dominant and rises, in accordance with linear stability analysis predicting that this is the most unstable mode, then quickly reaches a peak before collapsing as higher modes strengthen. In particular it becomes lower than the amplitudes of modes $n_{\theta}=1$ and $n_{\theta}=2$ around $z=r_{0}$. Between that position and $z \simeq 3.5 r_{0}$, in the flow region where mixing-layer roll-up and the first stage of vortex pairings occur, mode $n_{\theta}=1$, however, clearly dominates. Farther downstream, as turbulence is three-dimensional according to figure 13, the contribution of small-scale structures exceeds the others. In the tripped jets, the first three azimuthal modes weaken considerably with $u_{e}^{\prime} / u_{j}$, and high-order modes predominate over the entire mixing layers. At $z=r_{0}$ for instance, Fourier coefficients $\left.a_{u u}^{(\theta)}\right|_{n_{\theta}}$ for $n_{\theta}=1$ and $n_{\theta}=2$ are around 0.06 in Jet3\% and 0.01 in Jet6\%, and lower than 0.01 in Jet9\%. This demonstrates the gradual disappearance of large-scale structures in these jets. Note that the decrease of the contribution of modes $n_{\theta} \geqslant 21$ with the axial distance is due to the mixing-layer thickening, which straightforwardly leads to lower azimuthal modes. 


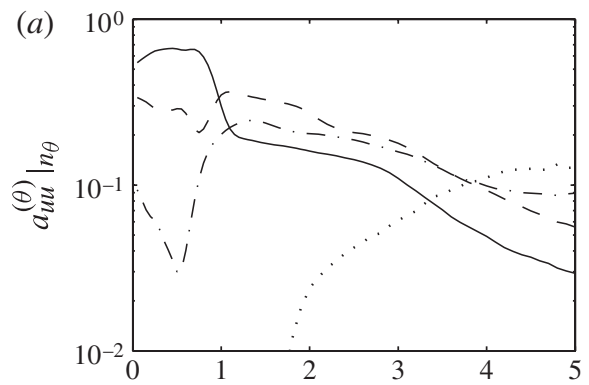

(b)
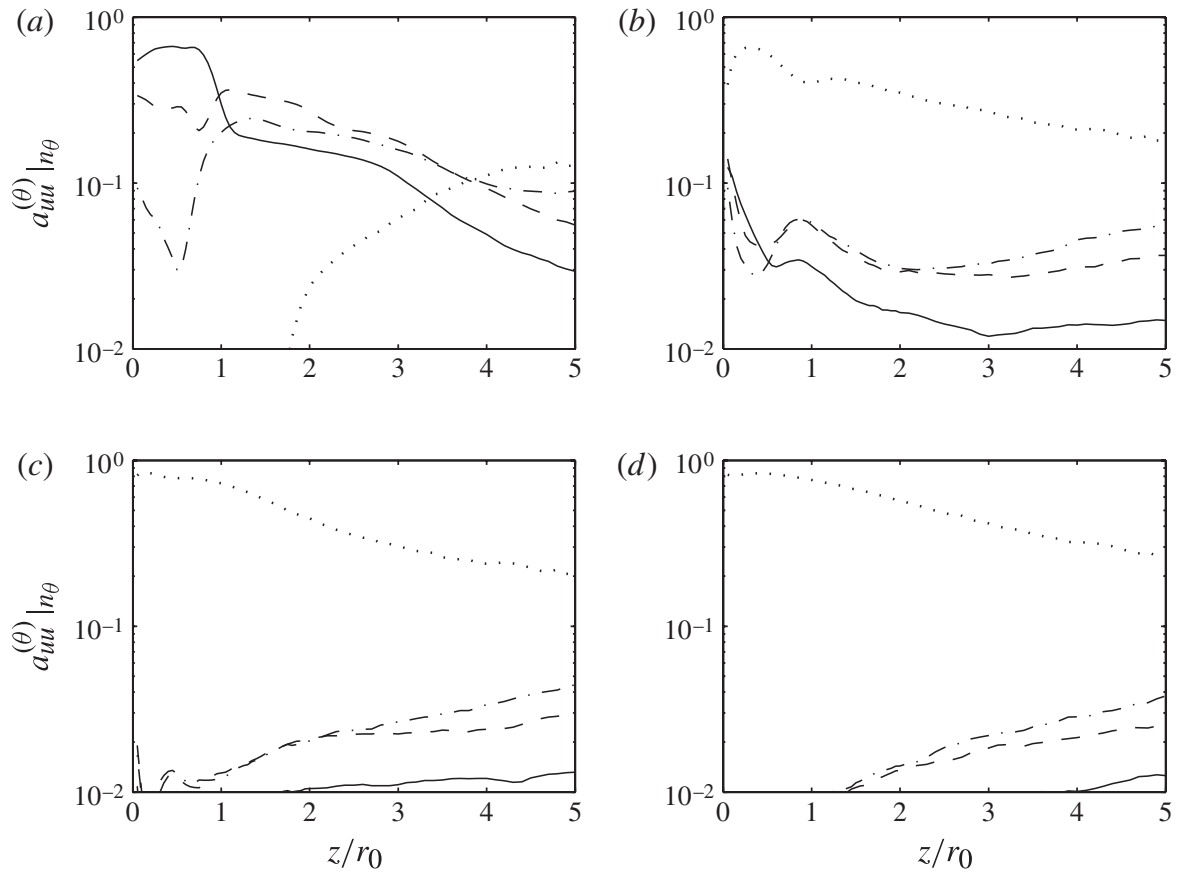

FIGURE 16. Variations of the coefficients $\left.a_{u u}^{(\theta)}\right|_{n_{\theta}}$ obtained from the cross-correlation functions $\mathscr{R}_{u u}^{(\theta)}$ of velocity $u_{z}^{\prime}$ at $r=r_{0}$ in $(a)$ Jet0\%, (b) Jet3\%, (c) Jet6\%, and (d) Jet9\%: ,$- n_{\theta}=0 ;---, n_{\theta}=1 ;-\cdot-\cdot, n_{\theta}=2 ; \ldots . . .$, sum for $n_{\theta} \geqslant 21$.

\subsubsection{Intermittency}

The turbulent phenomena taking place in the mixing layers of the jets are finally investigated through intermittency. In round jets, intermittency is known to be high at the interfaces between turbulent and non-turbulent zones, as shown by Chevray \& Tutu (1978) for instance. It can also be found to be significant on the centreline, which was attributed to the formation and evolution of ring vortices in the shear layers in Camussi \& Guj (1999), and to the merging of turbulent structures at the end of the potential core in Bogey et al. (2003, 2009a,b) and Bogey \& Bailly (2007). It was examined in mixing layers, and related to entrainment by Winant \& Browand (1974) and Briggs et al. (1996), among others. In the present study, as indicators of intermittency, skewness and kurtosis factors, defined as $\left.S\right|_{u_{z}^{\prime}}=\left\langle u_{z}^{\prime 3}\right\rangle /\left\langle u_{z}^{\prime 2}\right\rangle^{3 / 2}$ and $\left.K\right|_{u_{z}^{\prime}}=\left\langle u_{z}^{\prime 4}\right\rangle /\left\langle u_{z}^{\prime 2}\right\rangle^{2}$ for the axial velocity for example, are calculated at $r=r_{0}$. Skewness may in addition provide information on the effects of the large scales as argued in Mathis et al. (2011).

The skewness factors of axial and radial velocities $u_{z}^{\prime}$ and $u_{r}^{\prime}$ at $r=r_{0}$ are presented in figure 17. In Jet0\%, they respectively exhibit large positive and negative values around $z=r_{0}$, which can be attributed to the shear-layer roll-up. On the lip line, characterized initially by low-velocity laminar flow conditions, the generation of vortices therefore appears to intermittently induce strong bursts of high axial velocity, and downward penetration of fluid, hence entrainment. Farther downstream, similar deviations are observed again for the skewness factors of $u_{z}^{\prime}$ and $u_{r}^{\prime}$ around $z=2.8 r_{0}$, where the first stage of vortex pairings occurs. They are however much smaller, certainly because of the appearance of three-dimensional turbulence. The variations of 

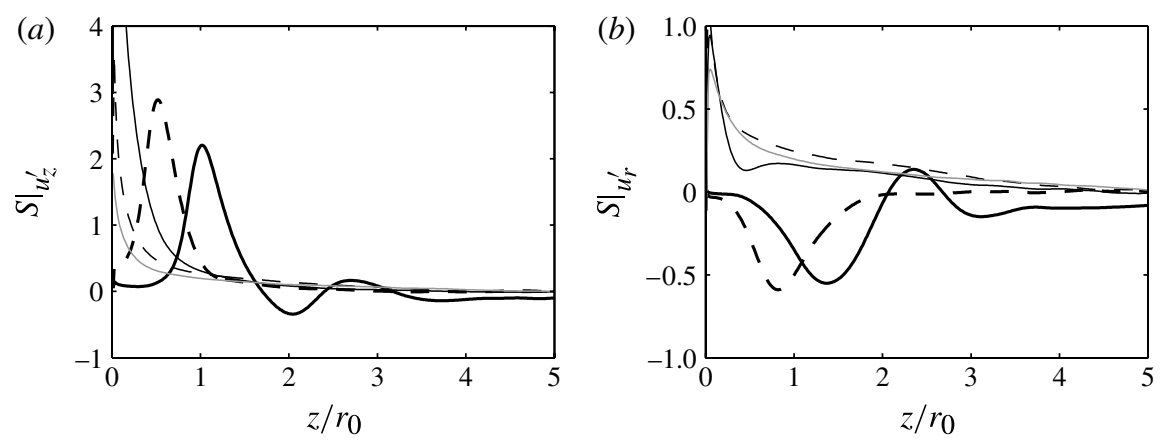

FIGURE 17. Variations of the skewness factor $S$ of fluctuating axial and radial velocities (a) $u_{z}^{\prime}$ and $(b) u_{r}^{\prime}$ at $r=r_{0}$ : - Jet0\%; ---, Jet3\%; - , Jet6\%;---, Jet9\%; - , Jet $12 \%$.

the skewness factors in Jet3\% resemble those in Jet0\%, indicating in particular the shear-layer rolling up slightly earlier in that case. In the three other jets, the results change significantly. The skewness factors of both fluctuating velocities are highly positive very near $z=0$, which most probably results from the rapid upward motions of turbulent disturbances coming from the boundary-layer profiles, then they quickly tend towards zero with increasing axial position as fully developed turbulence is found at $r=r_{0}$. The decrease of $\left.S\right|_{u_{r}^{\prime}}$ is moreover non-monotonic with a minimum around $z=0.4 r_{0}$ in Jet6\%, but monotonic in Jet9\% and Jet12\%, which suggests a weak shear-layer roll-up in the first jet, but no roll-up in the two other, highly tripped jets.

To further quantify the intermittency evidenced by the velocity skewness factors in the mixing layers, the kurtosis factors of fluctuating velocities $u_{z}^{\prime}, u_{r}^{\prime}$ and $u_{\theta}^{\prime}$, and vorticity norm $|\omega|^{\prime}$ at $r=r_{0}$ are displayed in figure 18. Large values of kurtosis, implying that the probability of having values far from the mean is strong, are generally obtained where skewness is significant. This is clearly the case in Jet0\% and Jet3\% around the shear-layer roll-up position but also around the vortex-pairing position, see in Jet0\% for instance the peaks of $\left.K\right|_{u_{z^{\prime}}}$ and $\left.K\right|_{|\omega|^{\prime}}$ at $z \simeq r_{0}$ and $z \simeq 2.8 r_{0}$, respectively. High kurtosis factors are also found in the jets with $u_{e}^{\prime} / u_{j} \geqslant 6 \%$ just downstream of the nozzle for all velocity components and vorticity. This is most likely due to the fact that the lip line in this region here lies at the interface between the initially highly disturbed shear layers and the laminar flow field surrounding the jets.

\subsection{Jet flow development}

\subsubsection{Vorticity fields}

Snapshots of the vorticity norm calculated up to $z=25 r_{0}$ are represented in figure 19 and supplementary movie 3 . With higher turbulence intensities at the nozzle exit, the mixing layers are seen to merge farther downstream, in agreement with the reduction in shear-layer growth rate described in the previous section, which leads to longer potential cores. Compare for instance figures 19(a), 19(d) and 19(e): the end of the potential core is around $z=10 r_{0}$ in Jet $0 \%$ but around $z=15 r_{0}$ in Jet $9 \%$ and Jet12\%.

\subsubsection{Mean and turbulent velocity profiles}

The variations of the centreline mean axial velocity $u_{c}$ and of the jet half-width $\delta_{0.5}$ are shown in figures $20(a)$ and $20(b)$. As the nozzle-exit turbulence intensity rises, the velocity decay and the jet spreading both start at increasing axial positions, leading 

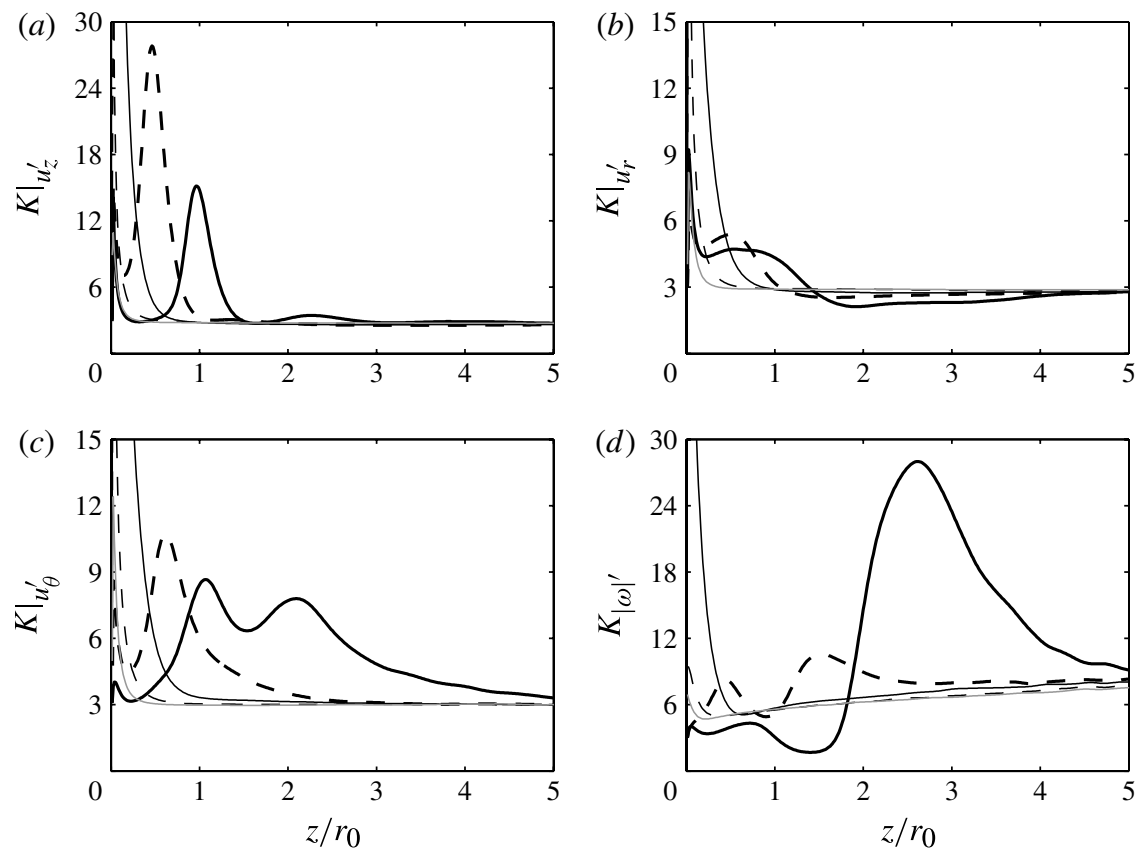

FIGURE 18. Variations of the kurtosis factor $K$ of fluctuating velocities $(a) u_{z}^{\prime}$, (b) $u_{r}^{\prime}$ and (c) $u_{\theta}^{\prime}$, and $(d)$ of the fluctuations of vorticity norm $|\omega|$, at $r=r_{0}$ : - Jet $0 \%$; ---, Jet3\%; —, Jet6\%; -- - Jet9\%; —- Jet12\%.

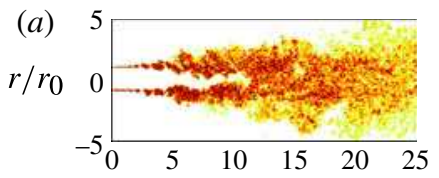

(b)

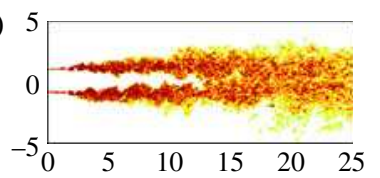

(c)

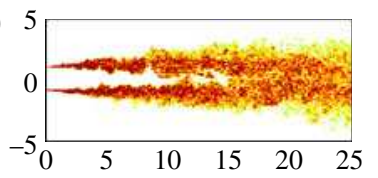

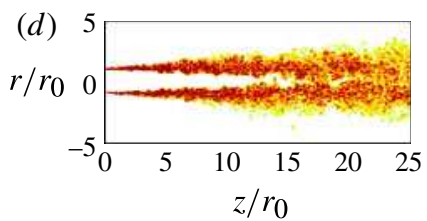

(e)

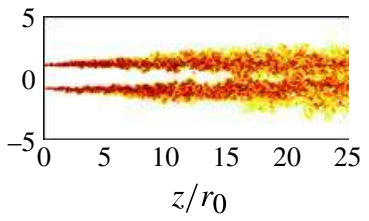

FIGURE 19. (Colour online) Snapshots in the $(z, r)$ plane of vorticity norm $|\boldsymbol{\omega}|$ in the full jets up to $z=25 r_{0}$ : $(a) \mathrm{Jet} 0 \%,(b) \mathrm{Jet} 3 \%,(c) \mathrm{Jet} 6 \%$, (d) Jet9\%, (e) Jet12\%. The colour scale ranges up to the level of $5 u_{j} / r_{0}$.

to potential cores ending at $z_{c}=9.3 r_{0}$ in Jet0\%, 12.9 $r_{0}$ in Jet3\%, 14.1 $r_{0}$ in Jet6\%, $15.9 r_{0}$ in $\mathrm{Jet} 9 \%$, and $17 r_{0}$ in $\mathrm{Jet} 12 \%$, where $u_{c}\left(z_{c}\right)=0.95 u_{j}$, as reported in table 4 and illustrated in figure $23(a)$ below. The development of the jet mean flow field is therefore delayed. Downstream of the potential core, it appears more rapid in Jet0\% than in the tripped jets. Similar discrepancies between tripped and untripped jets have been found experimentally by Russ \& Strykowski (1993), Raman et al. (1994) and Xu \& Antonia (2002). In order to provide more quantitative comparisons, measurements obtained by Lau, Morris \& Fisher (1979), Arakeri et al. (2003) and Fleury et al. 

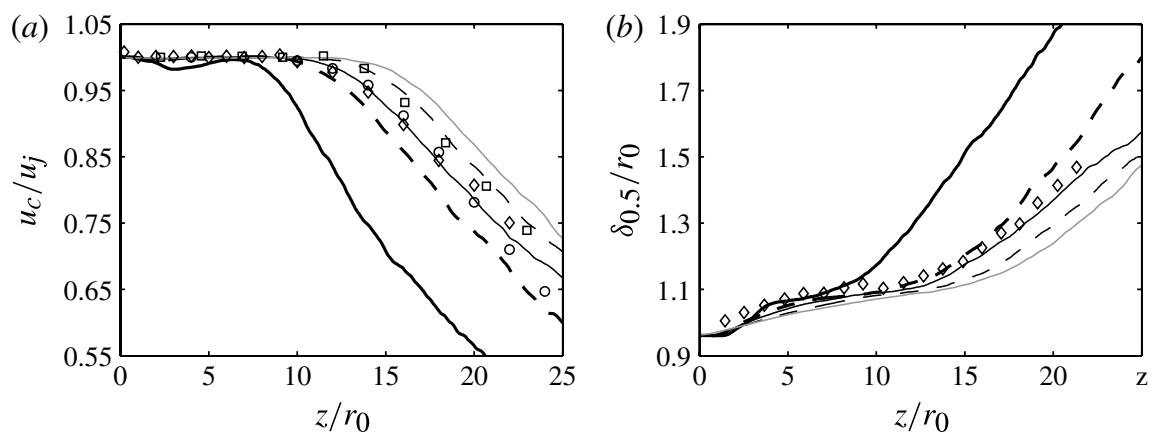

FIGURE 20. Variations $(a)$ of centreline mean axial velocity $u_{c}$ and $(b)$ of jet half-width $\delta_{0.5}$ : —, Jet0\%; ---, Jet3\%; — , Jet6\%; ---, Jet $9 \%$; —- Jet12\%. Measurements for Mach 0.9 jets at $R e_{D} \geqslant 5 \times 10^{5}: \square$, Arakeri et al. (2003); o, Lau et al. (1979); $\diamond$, Fleury et al. (2008).

$\begin{array}{lccc} & z_{c} / r_{0} & \left\langle u_{z}^{\prime 2}\right\rangle^{1 / 2} / u_{j}(\%) & \left\langle u_{r}^{\prime 2}\right\rangle^{1 / 2} / u_{j}(\%) \\ \text { Jet0\% } & 9.3 & 16.1 & 12.5 \\ \text { Jet3\% } & 12.9 & 12.4 & 10 \\ \text { Jet6\% } & 14.1 & 12.4 & 9.3 \\ \text { Jet9\% } & 15.9 & 11.4 & 9.4 \\ \text { Jet12\% } & 17 & 11.5 & 9.2\end{array}$

TABLE 4. Axial position of the end of the potential core $z_{c}$, and peak r.m.s. values of fluctuating velocities $u_{z}^{\prime}$ and $u_{r}^{\prime}$ on the jet axis.

(2008) for Mach number 0.9 jets at Reynolds numbers $R e_{D} \geqslant 5 \times 10^{5}$, thus probably all containing high initial disturbance levels, are also depicted in figure 20. Even though the nozzle-exit conditions in the experimental jets certainly vary, it is interesting to note that these data correspond most favourably to the results from Jet6\% and Jet9\%.

The r.m.s. values of the axial and radial fluctuating velocities along the jet centreline are displayed in figures $21(a)$ and $21(b)$. For higher nozzle-exit turbulence levels, the peak intensity values are reached farther downstream, in agreement with the delay in mean flow field development. They are reduced for $u_{z}^{\prime}$ and $u_{r}^{\prime}$, respectively, from $16.1 \%$ and $12.5 \%$ in Jet $0 \%$ down to around $11.5 \%$ and $9.3 \%$ in Jet $9 \%$ and Jet12\%; refer to table 4 for their values in Jet3\% and Jet6\% and to figure 23(b) for a representation of their variations with $u_{e}^{\prime} / u_{j}$. The maximum r.m.s. velocities are however rather similar in the four tripped jets, indicating a weak influence of the initial turbulence for $u_{e}^{\prime} / u_{j} \geqslant 3 \%$. The centreline intensity profiles in this case can also be seen to compare roughly with the scattered experimental data obtained for Mach number 0.9 jets at $R e_{D} \geqslant 5 \times 10^{5}$.

The variations of the centreline mean axial velocity and r.m.s. radial velocity are finally re-plotted in figures $22(a)$ and $22(b)$ as a function of $z-z_{c}$, i.e. shifted in the axial direction with respect to the jet potential core length. As previously suggested by figures 20 and 21 , the velocity decay is more rapid and the turbulence intensities are higher for the initially fully laminar jet. For the tripped jets, however, the mean and r.m.s. velocity profiles are very similar, and those from Jet6\%, Jet9\% and Jet $12 \%$ are almost indistinguishable. They also agree well with the measurements for high- 

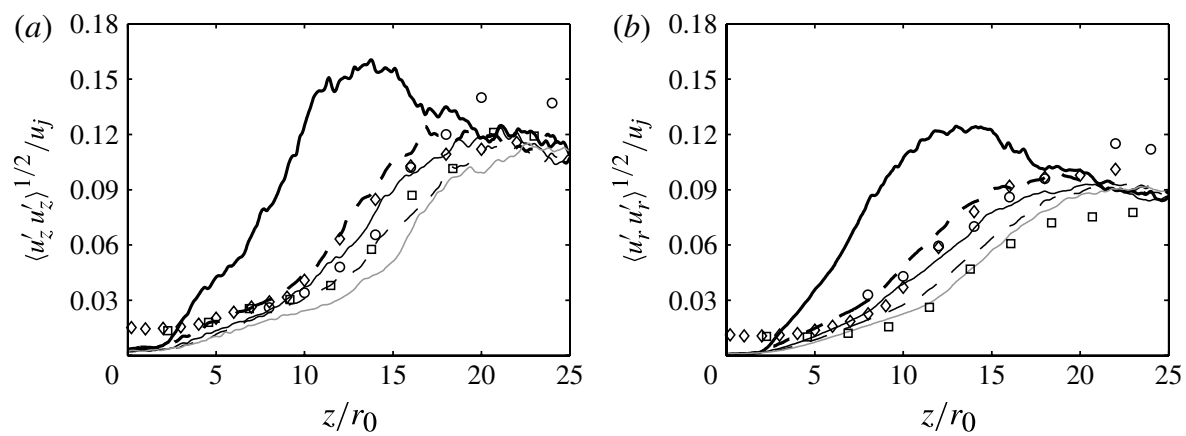

FIGURE 21. Variations of centreline r.m.s. values of fluctuating velocities $(a) u_{z}^{\prime}$ and $(b) u_{r}^{\prime}$ : —, Jet0\%; ---, Jet3\%; — , Jet6\%; ---, Jet9\%; — , Jet12\%. Measurements for Mach 0.9 jets at $R e_{D} \geqslant 5 \times 10^{5}: \square$, Arakeri et al. (2003); ○, Lau et al. (1979); $\diamond$, Fleury et al. (2008).
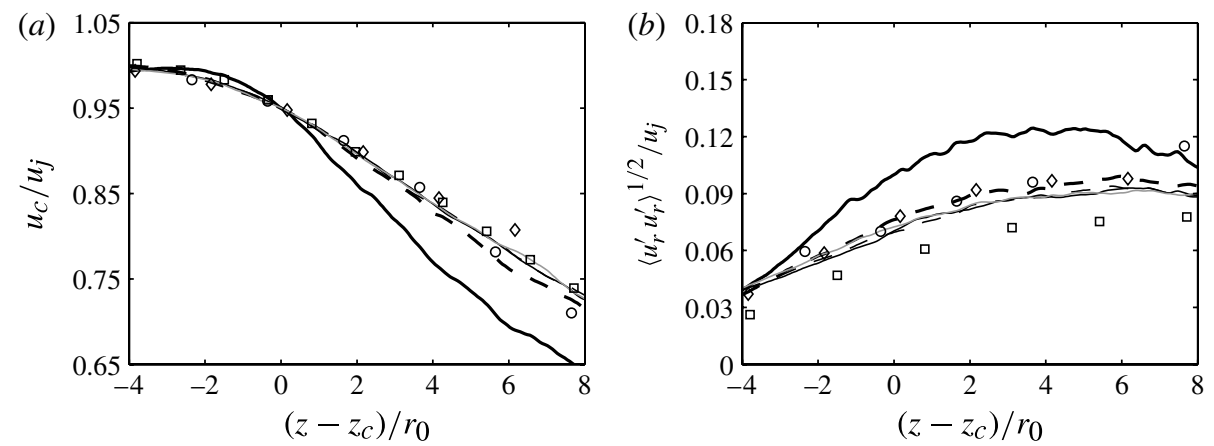

FIGURE 22. Variations $(a)$ of centreline mean axial velocity $u_{c}$, and $(b)$ of centreline r.m.s. values of radial velocity $u_{r}^{\prime}$, as a function of $\left(z-z_{c}\right) / r_{0}:-$, Jet $0 \% ;---$, Jet3\%; - Jet6\%; --- , Jet9\%; - Jet12\%. Measurements: $\square$, Arakeri et al. (2003); o, Lau et al. (1979); $\diamond$, Fleury et al. (2008).

Reynolds-number jets represented using the same axis scaling. Comparable results are found for the jet half-width and the centreline r.m.s. axial velocity. They are not shown here for the sake of brevity.

Except for the lengthening of the potential core, see figure 23, the impact of the initial turbulence intensity on the overall jet flow fields consequently appears relatively limited. For $u_{e}^{\prime} / u_{j} \geqslant 3 \%$, in particular, the flow field development downstream of the jet core is merely delayed without further notable change.

\subsection{Acoustic fields}

\subsubsection{Near-field pressure}

In order to provide a first glimpse into the jet acoustic features, snapshots of the pressure obtained directly in the LES are presented in figure 24 and supplementary movie 4. The sound fields are observed to depend significantly on the state of the boundary layers at the nozzle exit, in agreement with the findings of MolloChristensen et al. (1964), Zaman (1985b), Bridges \& Hussain (1987), Lilley (1994) and Harper-Bourne (2010), to mention a few well-established scientists in the field. As the exit turbulence intensity increases, the noise levels first appear to decrease 

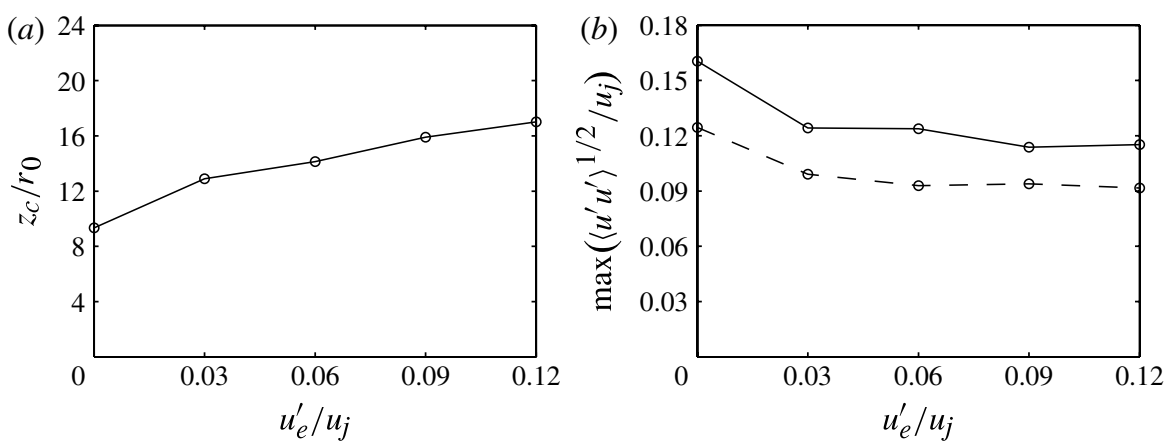

FIGURE 23. Variations with the exit turbulence level $u_{e}^{\prime} / u_{j},(a)$ of the axial position of the end of the potential core $z_{c}$, and $(b)$ of the peak r.m.s. values of velocities,$- u_{z}^{\prime}$ and,$--- u_{r}^{\prime}$ on the jet axis.
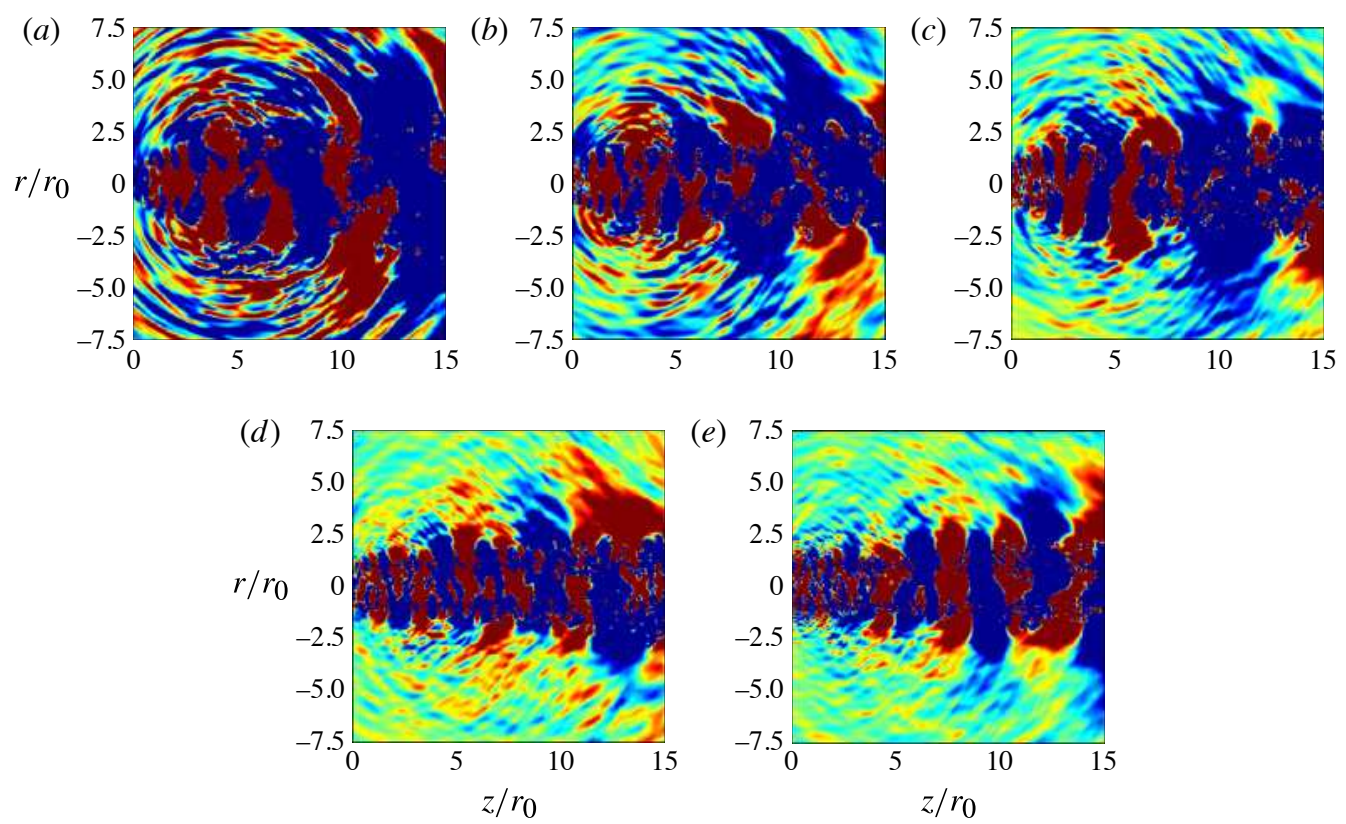

FIGURE 24. (Colour online) Snapshots in the $(z, r)$ plane of pressure $p-p_{\text {amb }}$ obtained by LES: $(a) \mathrm{Jet} 0 \%,(b) \mathrm{Jet} 3 \%,(c) \mathrm{Jet} 6 \%,(d) \mathrm{Jet} 9 \%,(e)$ Jet12\%. The colour scale ranges from -100 to $100 \mathrm{~Pa}$.

dramatically. The peak values of pressure fluctuations are for instance around $300 \mathrm{~Pa}$ in figure 24(a) for the initially fully laminar jet, while they are only around $50 \mathrm{~Pa}$ in figures $24(d)$ and $24(e)$ for the jets with $u_{e}^{\prime} / u_{j} \simeq 10 \%$. The structure of the sound fields is also seen to be modified. Acoustic waves are indeed clearly generated in the shear layers around $z=5 r_{0}$ in Jet0\%, whereas they show a more complex pattern for the initially highly disturbed jets.

\subsubsection{Far-field acoustic properties}

The quantitative and qualitative changes in the acoustic far fields are examined from the pressure signals obtained at 60 radii from the nozzle exit using the wave 


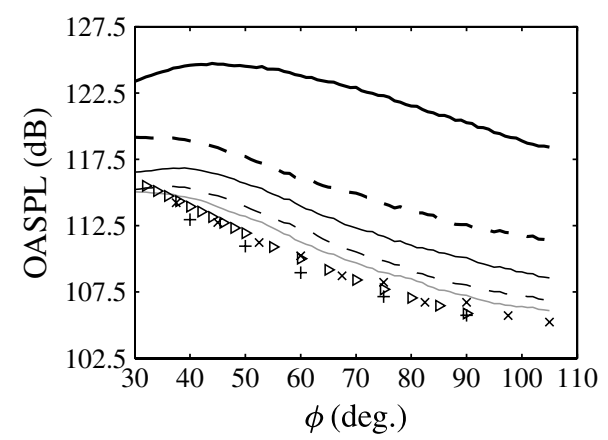

FIGURE 25. Overall sound pressure levels (OASPL) at $60 r_{0}$ from the pipe exit, as a function of the angle $\phi$ relative to the jet direction: —- Jet0\%; -- , Jet3\%; - Jet6\%; ---, Jet9\%; - Jet $12 \%$. Measurements for jets at $\operatorname{Re}_{D} \geqslant 5 \times 10^{5}:+$, Mollo-Christensen et al. (1964); $\times$, Lush (1971); $\triangleright$, Bogey et al. (2007).

extrapolation method documented in $\S 2.4$. The sound pressure levels determined at this distance are represented in figure 25 for angles $\phi$ between $30^{\circ}$ and $110^{\circ}$ relative to the flow direction. They are seen to decrease considerably with the nozzle-exit turbulence intensity for all angles. At $\phi=90^{\circ}$, in particular, the noise level is lower by $7.6 \mathrm{~dB}$ in Jet3\%, $10.1 \mathrm{~dB}$ in Jet6\%, $12.2 \mathrm{~dB}$ in Jet9\% and $12.9 \mathrm{~dB}$ in Jet12\% with respect to the level obtained for the untripped case. Similar observations have been made in experiments on circular jets at moderate Reynolds numbers with laminar or turbulent initial conditions. Zaman (1985b) for example reported a noise reduction of $\sim 4 \mathrm{~dB}$ between an untripped jet and a tripped jet at $R e_{D} \simeq 10^{5}$, in which the exit boundary-layer conditions were $R e_{\theta}=330$ and $u_{e}^{\prime} / u_{j}<1 \%$, and $R e_{\theta}=900$ and $u_{e}^{\prime} / u_{j} \simeq 9 \%$, respectively. In the same way, Bridges \& Hussain (1987) were able to demonstrate that, for two tripped/untripped jets at $R e_{D}=1.4 \times 10^{5}$, the initially laminar jet with $u_{e}^{\prime} / u_{j} \simeq 0.5 \%$ is $2.5 \mathrm{~dB}$ louder than the initially turbulent jet. In our previous LES of Mach number 0.9 round jets, the far-field noise levels at $\phi=90^{\circ}$ were also found to differ by $3 \mathrm{~dB}$ in Bogey et al. (2008) for two jets at $\operatorname{Re}_{D}=5 \times 10^{5}$ with $u_{e}^{\prime} / u_{j}=1.6$ or $9 \%$, and by $8 \mathrm{~dB}$ in Bogey \& Bailly (2010) for two jets at $R e_{D}=10^{5}$ with $u_{e}^{\prime} / u_{j}=0.3 \%$ or $1.9 \%$.

The sound pressure levels are also compared in figure 25 to those measured by Mollo-Christensen et al. (1964), Lush (1971) and Bogey et al. (2007) for Mach number 0.9 jets at $R e_{D} \geqslant 5 \times 10^{5}$. For all radiation angles, a substantial overestimation is observed for the jet with fully laminar initial conditions. The agreement between LES and experimental levels becomes much better as the nozzle-exit turbulence intensity increases. This tendency is illustrated in figure 26 for the angles $\phi=40^{\circ}$ and $90^{\circ}$. At $\phi=90^{\circ}$ for instance, the discrepancy with respect to the measurements is around $4 \mathrm{~dB}$ for Jet $6 \%, 2 \mathrm{~dB}$ for Jet $9 \%$ and only $1 \mathrm{~dB}$ for Jet $12 \%$. Therefore, as large-scale structures are weakened in the shear layers of the jets at $\operatorname{Re}_{D}=10^{5}$, the sound levels decrease asymptotically down to those found for jets at high Reynolds numbers $R e_{D} \geqslant 5 \times 10^{5}$, which are most likely to be initially turbulent and to emit negligible vortex pairing noise according to Hussain (1983) and Bridges \& Hussain (1987). Regarding the persistence of pairing-noise components of low intensity in Jet9\% and Jet12\%, suggested by the slightly higher sound levels with respect to the measurements in these two cases, it can be noted that for jets with exit parameters $R e_{D}, R e_{\theta}$ and $u_{e}^{\prime} / u_{j}$ identical to those in Jet9\%, Zaman (1985b) also obtained a notable 


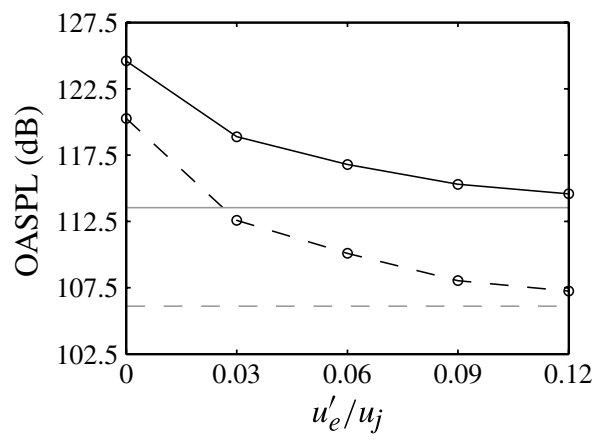

FIGURE 26. Variations with the exit turbulence level $u_{e}^{\prime} / u_{j}$ of the OASPL at $60 r_{0}$ from the pipe exit at the radiation angles: —,$\phi=40^{\circ} ;---, \phi=90^{\circ}$. Average values of the measurements by Mollo-Christensen et al. (1964), Lush (1971) and Bogey et al. (2007) for jets at $R e_{D} \geqslant 5 \times 10^{5}$ at:,$- \phi=40^{\circ} ;---, \phi=90^{\circ}$.

deviation compared to the levels extrapolated from high-Reynolds-number data using classical velocity power laws. As previously mentioned in Bogey et al. (2011a), this may be due to the combined effects of physical factors such as the moderate Reynolds number and the shear-layer thickness. Large-scale structures may in particular exist, albeit strongly attenuated, in highly disturbed or turbulent mixing layers at $\operatorname{Re}_{\theta}=900$; refer to recent results in Bogey et al. (2012).

The pressure spectra obtained at $60 r_{0}$ from the nozzle exit at the angles $\phi=30^{\circ}$, $40^{\circ}, 60^{\circ}$ and $90^{\circ}$ relative to the jet direction are represented in figure 27 , together with experimental spectra of Tanna (1977) and Bogey et al. (2007) for Mach number 0.9 jets at $R e_{D} \geqslant 7.8 \times 10^{5}$. For the initially fully laminar jet, the discrepancies with respect to the measurements are significant for both spectrum level and shape. At $\phi=30^{\circ}$, a peak is noticed around $S t_{D}=0.8$, which corresponds to $S t_{\theta}=0.007$, and was identified as the frequency of the first vortex pairings in the shear layers from figure $15(b)$ in $\$ 3.2 .3$. At the same angle, the acoustic components around $S t_{D} \simeq 0.15$ seem also strengthened, as was the case in Bogey \& Bailly (2010) for untripped jets at $R e_{D}=10^{5}$ with $\delta_{\theta}(0)=0.023 r_{0}$ and $0.012 r_{0}$. The amplification of the downstream-dominant low-frequency noise component certainly results here from the presence of a thick laminar shear layer, leading to a laminar-turbulent transition occurring in the vicinity of the end of the jet core, and hence to an increase in the r.m.s. velocity fluctuations in this region as depicted in figure 21 . With rising initial turbulence levels, in agreement with the trends exhibited in figure 25 , the sound spectra in figure 27 become progressively closer to the high- $R e_{D}$ experimental spectra at the four radiation angles. For Jet $9 \%$ and in Jet12\%, a good agreement with the measurements is thus achieved for Strouhal numbers lower than $S t_{D}=0.6$. A slight overestimation by $2-3 \mathrm{~dB}$ nevertheless persists for $S t_{D}>0.6$, which may be attributed to interactions of weak large-scale structures in the shear layers as discussed above.

The characteristics of the noise generated in the mixing layers are investigated in more detail based on the pressure signals at $60 r_{0}$ high-pass filtered to remove components below Strouhal number $S t_{D}=0.4$, which is half the vortex-pairing frequency. The cross-correlation functions $\mathscr{R}_{p p}^{(\theta)}$ of the pressure signals are first calculated in the azimuthal direction, following Maestrello (1976) for instance. The distribution of the acoustic energy with respect to mode $n_{\theta}$ is then determined by applying a Fourier series decomposition to $\mathscr{R}_{p p}^{(\theta)}$, yielding Fourier coefficients $\left.a_{p p}^{(\theta)}\right|_{n_{\theta}}$. 

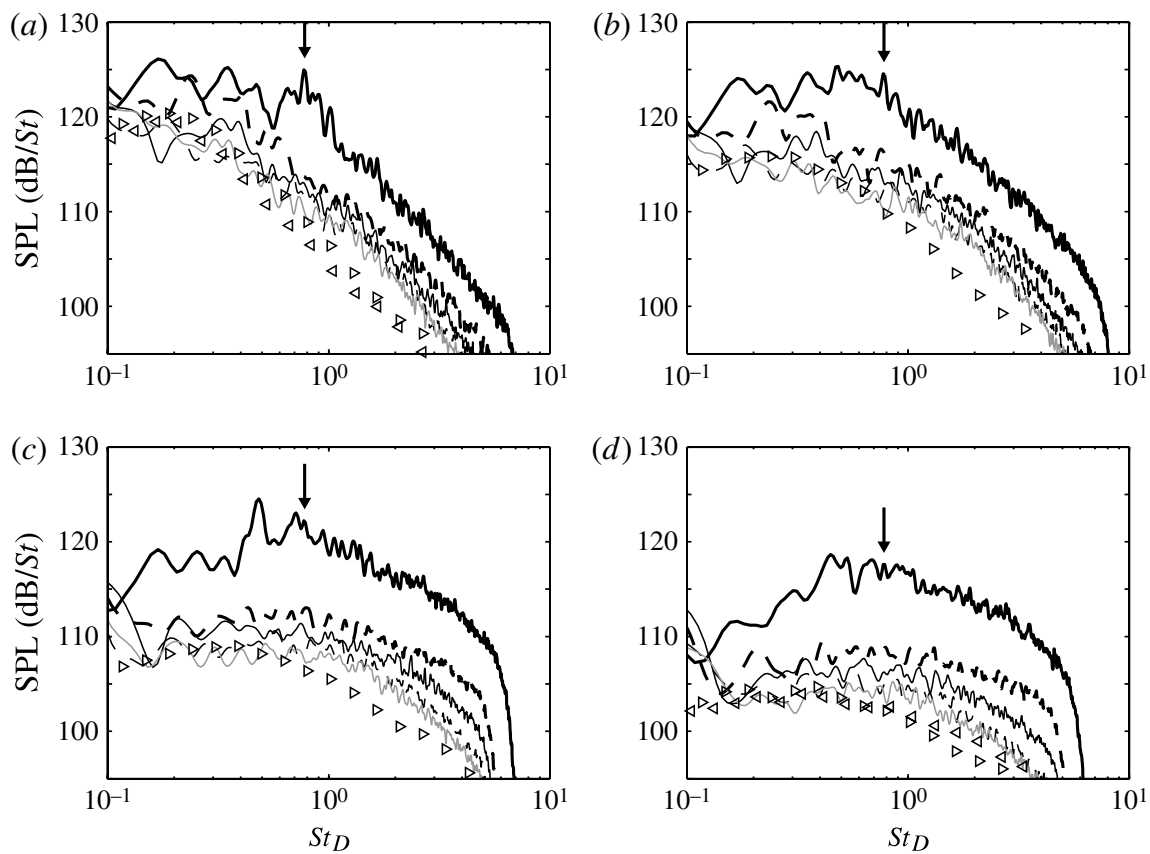

FIGURE 27. Sound pressure levels (SPL) at $60 r_{0}$ from the jet exit, as a function of $S t_{D}=f D / u_{j}$, at the angles $(a) \phi=30^{\circ},(b) 40^{\circ}$, (c) $60^{\circ}$ and $(d) 90^{\circ}:-$, Jet0\%; ---, Jet3\%; - Jet6\%; ---, Jet9\%; - Jet12\%. The arrow indicates $S t_{D}=0.8$. Measurements for jets at $\operatorname{Re}_{D} \geqslant 7.8 \times 10^{5}: \triangleleft$, Tanna (1977); $\triangleright$, Bogey et al. (2007).

The contributions of the first modes $n_{\theta}=0,1$ and 2 are thus presented in figure $28(a-c)$ as a function of the radiation angle $\phi$. They do not appear to change fundamentally with the nozzle-exit disturbance levels. For all jets, indeed, mode $n_{\theta}=1$ is dominant. Modes $n_{\theta}=0$ and 2 are also significant, but the relative amplitude of the former mode varies appreciably with angle $\phi$ whereas that of the latter does not. These trends are roughly similar to those exhibited by the Fourier coefficients estimated by Juvé \& Sunyach (1981) from far-field measurements filtered at $S t_{D}=1$ for a unexcited jet at $M=0.4$ and $R e_{D}=1.8 \times 10^{5}$. The Fourier coefficients provided by Juvé \& Sunyach (1981) are overall larger, which is not surprising because they are obtained for a single frequency, whereas the LES results are for the wide range of Strouhal numbers $S t_{D} \geqslant 0.4$, including higher-frequency components which undoubtedly have decorrelating effects. Furthermore, the Mach numbers differ, which may have an influence on the modal distribution of the sound fields as reported in Bogey \& Bailly (2006c). For the present jets, it is interesting to note that the contribution of mode $n_{\theta}=1$ to the far-field noise at $S t_{D} \geqslant 0.4$ is greater in the untripped case than in the tripped cases for $30^{\circ} \leqslant \phi \leqslant 90^{\circ}$. This can be due to the fact that the first vortex pairings in Jet0\% take place around $z=3 r_{0}$ in the mixing layers, where mode $n_{\theta}=1$ prevails according to figure $16(a)$ in $\S 3.2 .3$. The contribution of the axisymmetric mode is also higher in Jet0\% than in the other jets around $\phi=60^{\circ}$.

The contribution of higher azimuthal modes to the mixing-layer noise is now considered by displaying the sum of the Fourier coefficients $\left.a_{p p}^{(\theta)}\right|_{n_{\theta}}$ for $n_{\theta} \geqslant 9$ in figure $28(d)$. For all jets, it is negligible at $\phi=30^{\circ}$, but increases with the radiation angle to reach values around $10 \%$ at $\phi=90^{\circ}$. The values obtained for wide radiation 

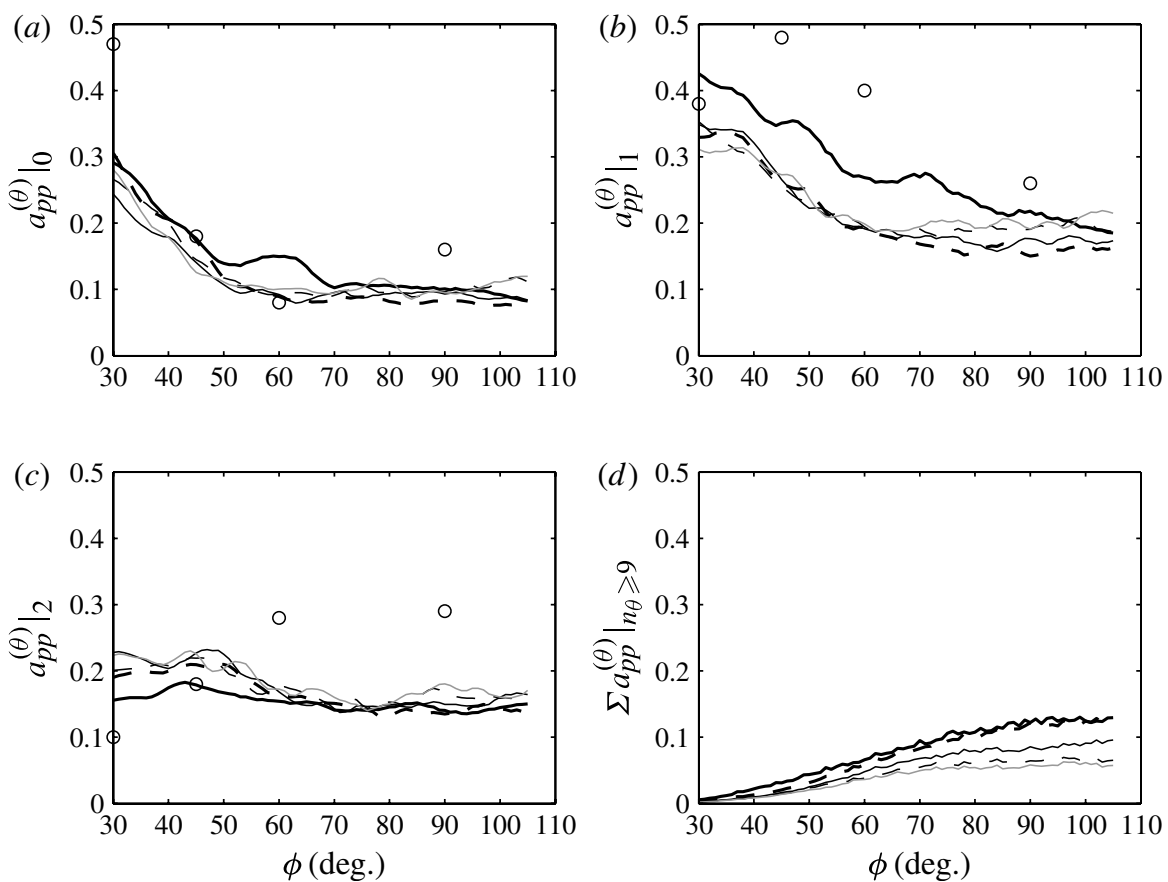

FIGURE 28. Coefficients $\left.a_{p p}^{(\theta)}\right|_{n_{\theta}}$ obtained from the cross-correlation functions $\mathscr{R}_{p p}^{(\theta)}$ of the fluctuating pressure at $60 r_{0}$ from the pipe exit, high-pass filtered for $S t_{D} \geqslant 0.4,(a) n_{\theta}=0$, (b) $n_{\theta}=1,(c) n_{\theta}=2$, and (d) sum for $n_{\theta} \geqslant 9$, as a function of the radiation angle $\phi$ : Jet0\%; ---, Jet3\%; ——, Jet6\%; ---, Jet9\%; - - Jet12\%; o, experimental data of Juvé \& Sunyach (1981) for the far field of a jet at $M=0.4$ and $R e_{D}=1.8 \times 10^{5}$ filtered at $S t_{D}=1$.

angles are moreover higher for the jets with lower nozzle-exit turbulence levels, in which stronger pairings of azimuthally-correlated vortices take place in the shear layers, which might appear contradictory. This result can however be explained by the downstream sound directivity of vortex pairing, which was shown by the simulations of Colonius, Lele \& Moin (1997) and Mitchell, Lele \& Moin (1999) for example, and is illustrated here by the fact that for Jet0\% the pairing frequency emerges more clearly for smaller radiation angles in the sound spectra of figure 27. At $\phi=90^{\circ}$ in particular, the spectrum is quite broad, and contains significant components at high Strouhal numbers. Vortex pairings therefore seem to lead to a broadband noise amplification relatively uniform in frequency, similarly to what was observed experimentally for excited jets at high Reynolds numbers; refer to the reviews of Crighton (1981) and Zaman (1985a) on that topic. This broadband noise can reasonably be believed to be generated in the mixing layers downstream of the first vortex pairings, where turbulent fluctuations, as revealed previously by figures 11 and 13, are intense and three-dimensional, thus enhancing the contributions of higher azimuthal modes to the far-field noise.

The importance of intermittency for noise generation in the present jets is finally studied, as was done previously by Juvé, Sunyach \& Comte-Bellot (1980), Bogey et al. (2003, 2009a,b), Bogey \& Bailly (2007), Hileman et al. (2005), or more recently by Cavalieri et al. (2010). For that purpose, the skewness and kurtosis factors of the far-field signals high-pass filtered for $S t_{D} \geqslant 0.4$ are computed, and presented in 
(a)

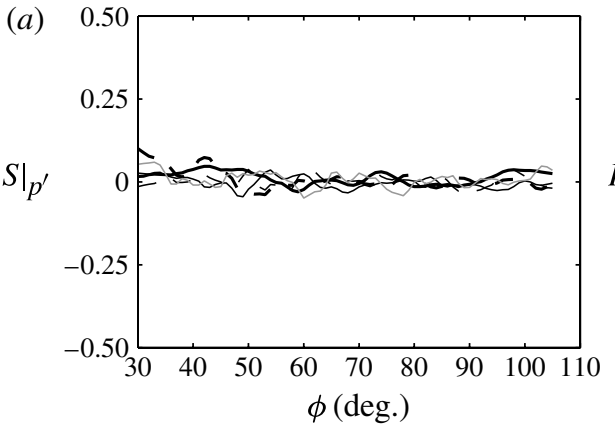

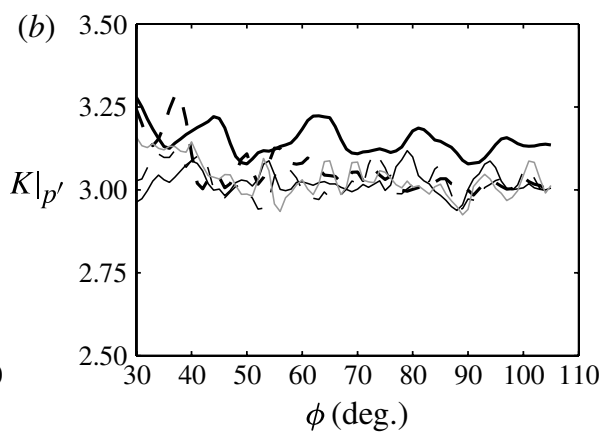

FIgURE 29. Skewness and kurtosis factors $(a) S$ and $(b) K$ of the fluctuating pressure at $60 r_{0}$ from the pipe exit, high-pass filtered for $S t_{D} \geqslant 0.4$, as a function of the radiation angle $\phi:-$ Jet0\%; ---, Jet3\%; —, Jet6\%;---, Jet9\%; —, Jet12\%.

figure 29 as a function of the radiation angle. Unlike what may be observed for supersonic jets as in Papamoschou \& Debiasi (2001) for instance, the deviations of the skewness factor with respect to zero are small in all cases, implying nearly symmetrical probability distributions for the pressure fluctuations. In the same way, the values taken by the kurtosis factor are generally close to the value of 3 expected for a Gaussian distribution. Larger values are however found for the untripped jet for all radiation angles, and for Jet $3 \%$ between $\phi=30^{\circ}$ and $\phi=40^{\circ}$, indicating appreciable intermittency of the acoustic waves in these cases. A further link is thus established between the turbulent events, namely shear-layer roll-up and vortex pairings, occurring intermittently in the mixing layers of the initially laminar jets according to figures 17 and 18 , and the strong far-field components radiated by these jets.

\section{Conclusion}

In this paper, the influence of the nozzle-exit disturbance level in round jets characterized by identical Mach and diameter-based Reynolds numbers and by effectively the same exit boundary-layer thicknesses is investigated. The effects on the initial turbulence, the shear layers, the jet development and the radiated sound field, as well as on the underlying physical mechanisms, are described in depth. They are found to be in good agreement with trends obtained in different experiments, most often dealing either with mixing layers, jet flows or noise, in which several initial flow parameters may vary. In this sense, to the best of our knowledge, the present work is the first one to study the impact, both hydrodynamic and acoustic, of nozzle-exit disturbance intensity on jets under such highly controlled conditions, hence to provide unambiguous results concerning the subject under consideration.

For the Mach number 0.9 jets at $R e_{D}=10^{5}$ and $R e_{\theta}=900$ examined here, a notable modification of the overall flow development with increasing initial turbulence level is the lengthening of the potential core. For $u_{e}^{\prime} / u_{j} \geqslant 3 \%$, nevertheless, the flow development downstream of the jet core remains nearly identical. The effects are much more spectacular on the shear-layer properties and consequently on noise features. For laminar initial conditions, the mixing layers are clearly dominated by linear instability waves just downstream of the nozzle exit, then by large-scale structures highly correlated in the azimuthal direction, whose intermittent pairings result in a rapid flow development and in high turbulence levels. As the nozzle-exit disturbance 
intensity increases, these structures become weaker, and three-dimensional turbulent features as well as high azimuthal modes tend to predominate in the entire mixing layers, leading to a slower shear-layer spreading and to a significant reduction of the r.m.s. velocity peak values, by a factor of $\sim 2$ between $u_{e}^{\prime} / u_{j}=0 \%$ and $u_{e}^{\prime} / u_{j} \simeq 10 \%$ for instance.

Simultaneously, for laminar jet exit conditions, strong acoustic components due to vortex pairings and to broadband noise amplification are generated respectively in the transitional and turbulent regions of the shear layers. With increasing $u_{e}^{\prime} / u_{j}$, their amplitudes become gradually lower, and the far-field sound levels decrease down to those measured for jets at Reynolds numbers $R e_{D} \geqslant 5 \times 10^{5}$. The reduction of the noise levels is observed to be sharp for low $u_{e}^{\prime} / u_{j}$, but smoother for higher $u_{e}^{\prime} / u_{j}$. At the radiation angle of $\phi=90^{\circ}$, for example, they differ by $7.6 \mathrm{~dB}$ between Jet $0 \%$ and $\mathrm{Jet} 3 \%, 2.5 \mathrm{~dB}$ between Jet3\% and Jet6\%, $1.9 \mathrm{~dB}$ between Jet6\% and Jet9\%, and only $0.7 \mathrm{~dB}$ between Jet9\% and Jet12\%.

These results emphasize the great importance of the initial disturbance level for the turbulent and noise generation mechanisms in subsonic round jets. Other parameters such as the nozzle-exit boundary-layer thickness, the momentum-thicknessbased Reynolds number $R e_{\theta}$, and the diameter-based Reynolds number $R e_{D}$, can be expected to also have major effects on the jet characteristics, especially regarding the persistence or suppression of large-scale structures in practical jets at Reynolds numbers typically around $10^{6}$, whose simulation would require many more grid points than the present LES. The influence of the Reynolds number was, for instance studied recently by Bogey et al. (2012) for tripped jets at moderate $R e_{D}$. Increasing the jet Reynolds number seems to induce changes similar to those obtained in this work when the nozzle-exit turbulence intensity rises.

\section{Acknowledgements}

This work was granted access to the HPC resources of the Institut du Développement et des Ressources en Informatique Scientifique (IDRIS) under the allocation 2011-020204 made by GENCI (Grand Equipement National de Calcul Intensif).

Supplementary movies are available at journals.cambridge.org/flm.

\section{Appendix. Amplification rates of linear instability modes downstream of the jet nozzle exit}

In this appendix, the amplification rates of instability waves in an axisymmetric mixing layer representative of the mean flows obtained downstream of the jet nozzle exit in the present study are examined based on a linear spatial stability analysis. Following Michalke (1984) for instance, an hyperbolic-tangent axisymmetric velocity profile $u_{z}(r) / u_{j}=\left[1+\tanh \left(\left(r_{0}-r\right) /\left(2 \delta_{\theta}\right)\right)\right] / 2$ is chosen, where $r_{0}$ is the radius and $\delta_{\theta}=0.018 r_{0}$ is the momentum thickness of the mixing layer, and the velocity $u_{j}$ provides a Mach number $M=u_{j} / c_{0}$ equal to 0.9. A generalized Rayleigh equation is solved to include the compressibility effects as done by Morris (1983). Viscous effects can moreover be expected to be very small at the Reynolds number $\operatorname{Re}_{\theta}=900$ considered in this paper according to the work of Morris (1976).

The dimensionless amplification rates $-\operatorname{Im}(k) \delta_{\theta}$, where $\operatorname{Im}(k)$ is the imaginary part of the wavenumber $k$, calculated for the azimuthal modes $n_{\theta}=0,1,2,3,4$ and 5 are presented in figure 30 as a function of Strouhal number $S t_{\theta}=f \delta_{\theta} / u_{j}$. In agreement with classical results of the literature, see the review by Michalke (1984), 


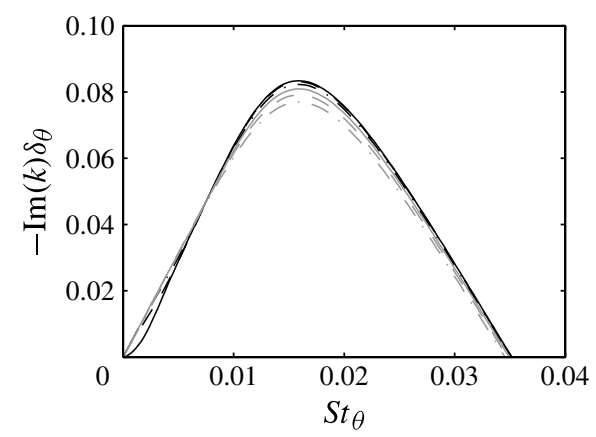

FIgURE 30. Amplification rates $-\operatorname{Im}(k) \delta_{\theta}$ as a function of Strouhal number $S t_{\theta}=f \delta_{\theta} / u_{j}$ of linear instability waves in an hyperbolic-tangent axisymmetric mixing layer at $M=0.9$ with $\delta_{\theta}=0.018 r_{0}$, for the azimuthal modes:,$- n_{\theta}=0 ;---, n_{\theta}=1 ;-\cdot-\cdot, n_{\theta}=2 ;-, n_{\theta}=3$; ,$--- n_{\theta}=4 ;-\cdot-\cdot, n_{\theta}=5$.

the axisymmetric mode $n_{\theta}=0$ is the least-stable mode, and reaches a maximum growth rate $-\operatorname{Im}(k) \delta_{\theta}=0.083$ around $S t_{\theta}=0.016$, corresponding well to the value $S t_{\theta}=0.0165$ found for a two-dimensional mixing layer. The amplification rates for the mode $n_{\theta}=1$ are very similar to those for the mode $n_{\theta}=0$. Then they gradually decrease for higher azimuthal modes. However, many modes appear nearly equally unstable, as was observed by Cohen \& Wygnanski (1987) in the mixing layer of a round jet with $\delta_{\theta}=0.013 r_{0}$. For the comparison with simulation results in $\$ 3.2 .3$, it can finally be noted that the amplification rates around $S t_{\theta}=0.014$ are close to $-\operatorname{Im}(k) \delta_{\theta}=0.08$ for the first three modes $n_{\theta}=0,1$ and 2 .

\section{REFERENCES}

Ahuja, K. K., Tester, B. J. \& TAnna, H. K. 1987 Calculation of far field jet noise spectra from near field measurements with true source location. J. Sound Vib. 116 (3), 415-426.

Arakeri, V. H., Krothapalli, A., Siddavaram, V., Alkislar, M. B. \& Lourenco, L. 2003 On the use of microjets to suppress turbulence in a Mach 0.9 axisymmetric jet. J. Fluid Mech. 490, 75-98.

Bailly, C. \& Bogey, C. 2004 Contributions of CAA to jet noise research and prediction. Intl J. Comput. Fluid Dyn. 18 (6), 481-491.

BATt, R. G. 1975 Some measurements on the effect of tripping the two-dimensional shear layer. AIAA J. 13 (2), 245-247.

Bell, J. H. \& MehtA, R. D. 1990 Development of a two-stream mixing layer from tripped and untripped boundary layers. AIAA J. 28 (12), 2034-2042.

Berland, J., Bogey, C., Marsden, O. \& Bailly, C. 2007 High-order, low dispersive and low dissipative explicit schemes for multi-scale and boundary problems. J. Comput. Phys. 224 (2), 637-662.

Bogey, C. \& Bailly, C. 2002 Three-dimensional non reflective boundary conditions for acoustic simulations: far-field formulation and validation test cases. Acta Acust. 88 (4), 463-471.

Bogey, C. \& BAilly, C. 2004 A family of low dispersive and low dissipative explicit schemes for flow and noise computations. J. Comput. Phys. 194 (1), 194-214.

Bogey, C. \& BAilly, C. $2006 a$ Large Eddy Simulations of transitional round jets: influence of the Reynolds number on flow development and energy dissipation. Phys. Fluids 18 (6), 065101.

Bogey, C. \& BAILly, C. $2006 b$ Large eddy simulations of round jets using explicit filtering with/without dynamic Smagorinsky model. Intl J. Heat Fluid Flow 27 (4), 603-610.

Bogey, C. \& BAILly, C. 2006c Investigation of downstream and sideline subsonic jet noise using large eddy simulation. Theor. Comput. Fluid Dyn. 20 (1), 23-40. 
Bogey, C. \& BAILly, C. 2007 An analysis of the correlations between the turbulent flow and the sound pressure field of subsonic jets. J. Fluid Mech. 583, 71-97.

Bogey, C. \& BAILly, C. 2009 Turbulence and energy budget in a self-preserving round jet: direct evaluation using large-eddy simulation. J. Fluid Mech. 627, 129-160.

Bogey, C. \& BAILly, C. 2010 Influence of nozzle-exit boundary-layer conditions on the flow and acoustic fields of initially laminar jets. J. Fluid Mech. 663, 507-539.

Bogey, C., BAIlly, C. \& JuvÉ, D. 2003 Noise investigation of a high subsonic, moderate Reynolds number jet using a compressible LES. Theor. Comput. Fluid Dyn. 16 (4), 273-297.

Bogey, C., BArRé, S. \& BAILly, C. 2008 Direct computation of the noise generated by subsonic jets originating from a straight pipe nozzle. Intl J. Aeroacoust. 7 (1), 1-22.

Bogey, C., Barré, S., Fleury, V., Bailly, C. \& Juvé, D. 2007 Experimental study of the spectral properties of near-field and far-field jet noise. Intl J. Aeroacoust. 6 (2), 73-92.

Bogey, C., Barré, S., Juvé, D. \& BAilly, C. $2009 a$ Simulation of a hot coaxial jet: direct noise prediction and flow-acoustics correlations. Phys. Fluids 21 (3), 035105.

Bogey, C., De Cacqueray, N. \& Bailly, C. 2009b A shock-capturing methodology based on adaptative spatial filtering for high-order nonlinear computations. J. Comput. Phys. 228 (5), $1447-1465$.

Bogey, C., De Cacqueray, N. \& Bailly, C. 2011 Finite differences for coarse azimuthal discretization and for reduction of effective resolution near origin of cylindrical flow equations. J. Comput. Phys. 230 (4), 1134-1146.

Bogey, C., Marsden, O. \& BAilly, C. $2011 a$ Large-eddy simulation of the flow and acoustic fields of a Reynolds number $10^{5}$ subsonic jet with tripped exit boundary layers. Phys. Fluids 23 (3), 035104.

Bogey, C., Marsden, O. \& BAILly, C. $2011 b$ On the spectra of nozzle-exit velocity disturbances in initially nominally turbulent, transitional jets. Phys. Fluids 23 (9), 091702.

Bogey, C., Marsden, O. \& Bailly, C. 2012 Flow and sound fields of initially tripped jets at Reynolds numbers ranging from 25,000 to 200,000. AIAA Paper 2012-1172. Meeting.

BRIDges, J. E. \& Hussain, A. K. M. F. 1987 Roles of initial conditions and vortex pairing in jet noise. J. Sound Vib. 117 (2), 289-311.

Briggs, D. A., Ferziger, J. H., Koseff, J. R. \& Monismith, S. G. 1996 Entrainment in a shear-free turbulent mixing layer. J. Fluid Mech. 310, 215-241.

Browand, F. K. \& LATigo, B. O. 1979 Growth of the two-dimensional mixing layer from a turbulent and nonturbulent boundary layer. Phys. Fluids 22 (6), 1011-1019.

Browand, F. K. \& TroutT, C. D. 1980 A note on spanwise structure in the two-dimensional mixing layer. J. Fluid Mech. 97 (4), 771-781.

Brown, G. L. \& Roshko, A. 1974 Density effect and large structure in turbulent mixing layers. J. Fluid Mech. 64, 775-816.

CAMussi, R. \& GuJ, G. 1999 Experimental analysis of intermittent coherent structures in the near field of a high Re turbulent jet flow. Phys. Fluids 11 (2), 423-431.

Cavalieri, A. V. G., Jordan, P., Gervais, Y., Wei, M. \& Freund, J. B. 2010 Intermittent sound generation and its control in a free-shear flow. Phys. Fluids 22 (11), 115113.

Chandrsuda, C., Mehta, R. D., Weir, A. D. \& Bradshaw, P. 1978 Effect of free stream turbulence on large structure in turbulent mixing layer. J. Fluid Mech. 85 (4), 693-704.

Chevray, R. \& TUtU, N. K. 1978 Intermittency and preferential transport of heat in a round jet. J. Fluid Mech. 88 (1), 133-160.

Cohen, J. \& Wygnanski, I. 1987 The evolution of instabilities in the axisymmetric jet. Part 1. The linear growth of disturbances near the nozzle. J. Fluid Mech. 176, 191-219.

Colonius, T. \& LELE, S. K. 2004 Computational aeroacoustics: progress on nonlinear problems of sound generation. Prog. Aero. 40, 345-416.

Colonius, T., Lele, S. K. \& Moin, P. 1997 Sound generation in a mixing layer. J. Fluid Mech. 330, 375-409.

Crighton, D. G. 1981 Acoustics as a branch of fluid mechanics. J. Fluid Mech. 106, 261-298.

Crow, S. C. \& Champagne, F. H. 1971 Orderly structure in jet turbulence. J. Fluid Mech. 48, $547-591$. 
Davies, P. O. A. L., Fisher, M. J. \& Barratt, M. J. 1963 The characteristics of the turbulence in the mixing region of a round jet. J. Fluid Mech. 15, 337-367.

Domaradzki, J. A. \& YeE, P. P. 2000 The subgrid-scale estimation model for high Reynolds number turbulence. Phys. Fluids 12 (1), 193-196.

Eggels, J. G. M., Unger, F., Weiss, M. H., Westerweel, J., Adrian, R. J., Friedrich, R. \& Nieuwstadt, F. T. M. 1994 Fully developed turbulent pipe flow: a comparison between direct numerical simulation and experiment. J. Fluid Mech. 268, 175-209.

Fleury, V., Bailly, C., Jondeau, E., Michard, M. \& Juvé, D. 2008 Space-time correlations in two subsonic jets using dual-PIV measurements. AIAA J. 46 (10), 2498-2509.

FREUND, J. B. 2001 Noise sources in a low-Reynolds-number turbulent jet at Mach 0.9. J. Fluid Mech. 438, 277-305.

Ghosh, S., Foysi, H. \& Friedrich, R. 2010 Compressible turbulent channel and pipe flow: similarities and differences. J. Fluid Mech. 648, 155-181.

Grosche, F.-R. 1974 Distributions of sound source intensities in subsonic and supersonic jets. AGARD-CP-131, 4-1 to 4-10.

Gutmark, E. \& Ho, C.-M. 1983 Preferred modes and the spreading rates of jets. Phys. Fluids 26 (10), 2932-2938.

HARper-Bourne, M. 2010 Jet noise measurements: past and present. Intl J. Aeroacoust. 9 (4 \& 5 ), 559-588.

Hileman, J. I., Thurow, B. S., Caraballo, E. J. \& Samimy, M. 2005 Large-scale structure evolution and sound emission in high speed jets: real-time visualization with simultaneous acoustic measurements. J. Fluid Mech. 544, 277-307.

Hill, W. G., Jenkins, R. C. \& Gilbert, B. L. 1976 Effects of the initial boundary-layer state on turbulent jet mixing. AIAA J. 14 (11), 1513-1514.

Ho, C.-M. \& Huerre, P. 1984 Perturbed free shear layers. Annu. Rev. Fluid Mech. 23 (3), $365-424$.

Husain, Z. D. \& Hussain, A. K. M. F. 1979 Axisymmetric mixing layer: influence of the initial and boundary conditions. AIAA J. 17 (1), 48-55.

Hussain, A. K. M. F. 1983 Coherent structures-reality and myth. Phys. Fluids 26 (10), 2816-2850.

Hussain, A. K. M. F. \& Husain, Z. D. 1980 Turbulence structure in the axisymmetric free mixing layer. AIAA J. 18 (12), 1462-1469.

Hussain, A. K. M. F \& ZEDAN, M. F. $1978 a$ Effects of the initial condition on the axisymmetric free shear layer: Effects of the initial momentum thickness. Phys. Fluids 21 (7), 1100-1112.

Hussain, A. K. M. F. \& ZedAn, M. F. $1978 b$ Effects of the initial condition on the axisymmetric free shear layer: Effects of the initial fluctuation level. Phys. Fluids 21 (9), 1475-1481.

Jones, B. G., Planchon, H. P. \& Hammersley, R. J. 1973 Turbulent correlation measurements in a two-stream mixing layer. AIAA J. 11 (8), 1146-1150.

JuVÉ, D. \& SUNYACH, M. 1981 Near and far field azimuthal correlations for excited jets. AIAA Paper 81-2011.

Juvé, D., Sunyach, M. \& Comte-Bellot, G. 1980 Intermittency of the noise emission in subsonic cold jets. J. Sound Vib. 71 (3), 319-332.

KIM, J. \& CHOI, H. 2009 Large eddy simulation of a circular jet: effect of inflow conditions on the near field. J. Fluid Mech. 620, 383-411.

LAU, J. C., Morris, P. J. \& Fisher, M. J. 1979 Measurements in subsonic and supersonic free jets using a laser velocimeter. J. Fluid Mech. 93 (1), 1-27.

LEPICOVSKY, J. \& BROWN, W. H. 1989 Effects of nozzle exit boundary-layer conditions on excitability of heated free jets. AIAA J. 27 (6), 712-718.

Lilley, G. M. 1994 Jet noise classical theory and experiments. In Aeroacoustics of Flight Vehicles (ed. H. H. Hubbard). Noise Sources, vol. 1. pp. 211-289. Acoustical Society of America.

Lush, P. A. 1971 Measurements of subsonic jet noise and comparison with theory. J. Fluid Mech. 46 (3), 477-500.

Maestrello, L. 1976 Two points correlations of sound pressure in the far field of a jet: Experiment. Tech. Mem. 72835. NASA-Langley Research Center. 
Maestrello, L. \& MCDAid, E. 1971 Acoustic characteristics of a high-subsonic jet. AIAA J. 9 (6), 1058-1066.

Mathis, R., Marusic, I., Hutchins, N. \& SReenivasan, K. R. 2011 The relationship between the velocity skewness and the amplitude modulation of the small scale by the large scale in turbulent boundary layers. Phys. Fluids 23 (12), 121702.

Michalke, A. 1984 Survey on jet instability theory. Prog. Aerosp. Sci. 21, 159-199.

Mitchell, B. E., Lele, S. K. \& Moin, P. 1999 Direct computation of the sound generated by vortex pairing in an axisymmetric jet. J. Fluid Mech. 383, 113-142.

Mohseni, K. \& Colonius, T. 2000 Numerical treatment of polar coordinate singularities. J. Comput. Phys. 157 (2), 787-795.

Mollo-Christensen, E., Kolpin, M. A. \& Martucelli, J. R. 1964 Experiments on jet flows and jet noise far-field spectra and directivity patterns. J. Fluid Mech. 18, 285-301.

Monty, J. P., Hutchins, N., NG, H. C. H., Marusic, I. \& Chong, M. S. 2009 A comparison of turbulent pipe, channel and boundary layer flows. J. Fluid Mech. 632, 431-442.

MORRIS, P. J. 1976 The spatial viscous instability of axisymmetric jets. J. Fluid Mech. 77 (3), 511-529.

MorRIS, P. J. 1983 Viscous stability of compressible axisymmetric jets. AIAA J. 21 (4), 481-482.

MORRIS, P. J. \& ZAMAN, K. B. M. Q. 2009 Velocity measurements in jets with application to noise source modelling. J. Sound Vib. 329 (4), 394-414.

Papamoschou, D. \& Debiasi, M. 2001 Directional suppression of noise from a high-speed jet. AIAA J. 39 (3), 380-387.

Raman, G., Rice, E. J. \& Reshotko, E. 1994 Mode spectra of natural disturbances in a circular jet and the effect of acoustic forcing. Exp. Fluids 17, 415-426.

Raman, G., Zaman, K. B. M. Q. \& Rice, E. J. 1989 Initial turbulence effect on jet evolution with and without tonal excitation. Phys. Fluids A 1 (7), 1240-1248.

RUSS, S. \& STRYKOWSKI, P. J. 1993 Turbulent structure and entrainment in heated jets: The effect of initial conditions. Phys. Fluids A 5 (12), 3216-3225.

SuzUKI, T. \& COLONIUS, T. 2007 Instability waves in a subsonic round jet detected using a near-field phased microphone array. J. Fluid Mech. 565, 197-226.

TAM, C. K. W. \& DONG, Z. 1996 Radiation and outflow boundary conditions for direct computation of acoustic and flow disturbances in a non-uniform mean flow. J. Comput. Acoust. 4 (2), 175-201.

TAnnA, H. K. 1977 An experimental study of jet noise. Part I: Turbulent mixing noise. J. Sound Vib. 50 (3), 405-428.

TOMkins, C. D. \& ADRIAN, R. J. 2003 Spanwise structure and scale growth in turbulent boundary layers. J. Fluid Mech. 490, 37-74.

TOMKINS, C. D. \& AdRIAN, R. J. 2005 Energetic spanwise modes in the logarithmic layer of a turbulent boundary layer. J. Fluid Mech. 545, 141-162.

Visbal, M. R. \& RizzetTA, D. P. 2002 Large-Eddy Simulation on curvilinear grids using compact differencing and filtering schemes. Trans. ASME: J. Fluids Engng 124 (4), 836-847.

Wang, M., Freund, J. B. \& Lele, S. K. 2006 Computational prediction of flow-generated sound. Annu. Rev. Fluid Mech. 38, 483-512.

Winant, C. D. \& BRowand, F. K. 1974 Vortex pairing: the mechanism od turbulent mixing-layer growth at moderate Reynolds number. J. Fluid Mech. 63 (2), 237-255.

Wygnanski, I., Oster, D., Fiedler, H. \& Dziomba, B. 1979 On the perseverance of a quasi-two-dimensional eddy-structure in a turbulent mixing layer. J. Fluid Mech. 93 (2), 325-335.

Xu, G. \& Antonia, R. A. 2002 Effects of different initial conditions on a turbulent free jet. Exp. Fluids 33, 677-683.

ZAMAN, K. B. M. Q. $1985 a$ Far-field noise of subsonic jet under controlled excitation. J. Fluid Mech. 152, 83-111.

ZAMAN, K. B. M. Q. $1985 b$ Effect of initial condition on subsonic jet noise. AIAA J. 23, 1370-1373.

ZAMAN, K. B. M. Q. \& Hussain, A. K. M. F. 1980 Vortex pairing in a circular jet under controlled excitation. Part 1. General jet response. J. Fluid Mech. 101 (3), 449-491. 